\title{
Bounding marginal densities via affine isoperimetry
}

\author{
Susanna Dann \\ Grigoris Paouris \\ Peter Pivovarov
}

June 22, 2018

\begin{abstract}
Let $\mu$ be a probability measure on $\mathbb{R}^{n}$ with a bounded density $f$. We prove that the marginals of $f$ on most subspaces are well-bounded. For product measures, studied recently by Rudelson and Vershynin, our results show there is a trade-off between the strength of such bounds and the probability with which they hold. Our proof rests on new affinely-invariant extremal inequalities for certain averages of $f$ on the Grassmannian and affine Grassmannian. These are motivated by Lutwak's dual affine quermassintegrals for convex sets. We show that key invariance properties of the latter, due to Grinberg, extend to families of functions. The inequalities we obtain can be viewed as functional analogues of results due to Busemann-Straus, Grinberg and Schneider. As an application, we show that without any additional assumptions on $\mu$, any marginal $\pi_{E}(\mu)$, or a small perturbation thereof, satisfies a nearly optimal small-ball probability.
\end{abstract}

\section{Introduction}

In this paper, we discuss connections between affine isoperimetric inequalities in convex geometry and concentration results for high dimensional probability distributions. We address the following question: if $\mu$ is a probability measure on $\mathbb{R}^{n}$ with a bounded density, to what extent are its marginal densities also bounded? Recall that if $\mu$ has density $f$ and $E$ is a $k$-dimensional subspace of $\mathbb{R}^{n}$, the density of the marginal $\pi_{E}(\mu)$ on $E$ is given by

$$
f_{\pi_{E}(\mu)}(x)=\int_{E^{\perp}+x} f(y) d y \quad(x \in E) .
$$

Rudelson and Vershynin [39] recently proved that if $f(x)=\prod_{i=1}^{n} f_{i}\left(x_{i}\right)$, where each $f_{i}$ is a bounded density on $\mathbb{R}$, then for every $1 \leqslant k \leqslant n$ and 
every $k$-dimensional subspace $E$,

$$
\left\|f_{\pi_{E}(\mu)}\right\|_{\infty}^{1 / k} \leqslant C \max _{i \leqslant n}\left\|f_{i}\right\|_{\infty}
$$

where $C$ is a numeric constant and $\|\cdot\|_{\infty}$ is the $L^{\infty}$-norm. On the other hand, even for products, the stronger inequality

$$
\left\|f_{\pi_{E}(\mu)}\right\|_{\infty}^{1 / k} \leqslant C\|f\|_{\infty}^{1 / n}
$$

need not hold for all subspaces $E$; indeed, if $f_{1}, \ldots, f_{k}$ are very peaked, the left-hand side of (1.3) can be arbitrarily large for the coordinate subspace $E=\operatorname{span}\left\{e_{1}, \ldots, e_{k}\right\}$, while the right-hand side can be well-behaved since

$$
\|f\|_{\infty}^{1 / n} \leqslant \sqrt[n]{\left\|f_{1}\right\|_{\infty} \cdots\left\|f_{n}\right\|_{\infty}} \leqslant \max _{i \leqslant n}\left\|f_{i}\right\|_{\infty} .
$$

Nevertheless, we show that for an arbitrary bounded density $f$, most of its marginals nearly satisfy (1.3), which we quantify with respect to the Haar probability measure $\mu_{n, k}$ on the Grassmannian manifold $G_{n, k}$ of all $k$-dimensional subspaces of $\mathbb{R}^{n}$.

Theorem 1.1. Let $\mu$ be a probability measure on $\mathbb{R}^{n}$ with a bounded density $f$. Then for each $1 \leqslant k \leqslant n-1$, there exists $\mathcal{A} \subseteq G_{n, k}$ with $\mu_{n, k}(\mathcal{A}) \geqslant 1-2 e^{-k n}$ such that for every $E \in \mathcal{A}$,

$$
f_{\pi_{E}(\mu)}(x)^{1 / k} \leqslant C\|f\|_{\infty}^{1 / n}
$$

for all $x \in E$, except possibly on a set of $\pi_{E}(\mu)$-measure less than $e^{-k n}$.

Thus given $f$, first sampling $E \in G_{n, k}$ according to $\mu_{n, k}$ and then $x \in E$ according to $\pi_{E}(\mu),(1.5)$ holds with overwhelming probability; on $G_{n, k}$ this is optimal (see Lemma 6.3). Furthermore, one must exclude exceptional sets of positive $\pi_{E}(\mu)$-measure as can be seen by considering a neighborhood of a Besicovitch set (see, e.g., [2, Chapter 9]).

As discussed in [39], bounds for marginals are connected to smallball probabilities, which are useful in random matrix theory e.g., [40]. If $X$ is a random vector in $\mathbb{R}^{n}$ with density $f$, then $f_{\pi_{E}(\mu)}$ gives the density of the orthogonal projection $P_{E} X$ of $X$ onto $E$. When $f$ is bounded, Theorem 1.1 implies that for every $E \in \mathcal{A}, \varepsilon>0$ and any $z \in E$,

$$
\mathbb{P}\left(\left|P_{E} X-z\right| \leqslant \varepsilon \sqrt{k}\right) \leqslant\left(C_{1} \varepsilon\|f\|_{\infty}^{1 / n}\right)^{k n /(n+1)},
$$


where $|\cdot|$ denotes the Euclidean norm. In contrast, (1.2) implies that for any $z \in E$

$$
\mathbb{P}\left(\left|P_{E} X-z\right| \leqslant \varepsilon \sqrt{k}\right) \leqslant\left(C_{2} \varepsilon \max _{i}\left\|f_{i}\right\|_{\infty}\right)^{k} .
$$

Thus if $f=\prod_{i} f_{i}$ and $\left\|f_{i}\right\|_{\infty}$ are not identical, the base in the probability in (1.6) is smaller than that in (1.7) (cf. (1.4)) but the exponent $\frac{n}{n+1}$ is slightly worse. Furthermore, for subspaces $E$ that do not belong to $\mathcal{A}$, one can perturb them to ensure that (1.6) holds (see Corollary 6.5).

The goal of this paper is to show that a purely probabilistic statement such as Theorem 1.1 is ultimately based on an affine-invariance property of certain integrals on the Grassmannian $G_{n, k}$ and affine Grassmannian $M_{n, k}$ and corresponding extremal inequalities. Here $M_{n, k}$ is equipped with its cannonical rigid-motion invariant measure $v_{n, k}$ (see Section 2). In particular, for non-negative, bounded integrable functions $f$ on $\mathbb{R}^{n}$, we consider

$$
\int_{G_{n, k}} \frac{\left(\int_{E} f(x) d x\right)^{n}}{\left\|\left.f\right|_{E}\right\|_{\infty}^{n-k}} d \mu_{n, k}(E)
$$

where $\left.f\right|_{E}$ is the restriction of $f$ to $E$, and

$$
\int_{M_{n, k}} \frac{\left(\int_{F} f(x) d x\right)^{n+1}}{\left\|\left.f\right|_{F}\right\|_{\infty}^{n-k}} d v_{n, k}(F) .
$$

Our interest in such quantities stems from the following notion: for $1 \leqslant k<n$, the dual affine quermassintegrals of a convex body $K \subset \mathbb{R}^{n}$ are defined by

$$
\widetilde{\Phi}_{n-k}(K)=\frac{\omega_{n}}{\omega_{k}}\left(\int_{G_{n, k}}|K \cap E|^{n} d \mu_{n, k}(E)\right)^{1 / n}
$$

where $\omega_{n}$ denotes the volume of the Euclidean ball $B_{2}^{n}$ in $\mathbb{R}^{n}$ of radius one and $|\cdot|$ denotes Lebesgue measure. These were introduced by Lutwak (see [26], [27] for background) and have proved to be an indespensable tool for quantitative questions concerning high-dimensional probability distributions, e.g., [29], [34], [35]. In [18], Grinberg proved that $\widetilde{\Phi}_{n-k}(K)=\widetilde{\Phi}_{n-k}(S K)$ for each volume-preserving linear transformation $S$. Motivated by Grinberg's result, we prove that the quantities in (1.8) and (1.9) are also invariant under volume preserving linear and affine transformations, respectively. Our argument uses the structure of semisimple Lie groups. 
In the case when $f=\mathbb{1}_{K}$, where $K$ is a convex body (or compact set), both (1.8) and (1.9) satisfy corresponding affine isoperimetric inequalities. In particular, a result of Busemann-Straus [8] and Grinberg [18] states that if $K$ is a convex body in $\mathbb{R}^{n}$ and $1 \leqslant k \leqslant n-1$, then

$$
\int_{G_{n, k}}|K \cap E|^{n} d \mu_{n, k}(E) \leqslant \frac{\omega_{k}^{n}}{\omega_{n}^{k}}|K|^{k} ;
$$

when $k>1$, equality holds only for origin-symmetric ellipsoids. The $k=n-1$ case is Busemann's seminal intersection inequality [7]. For the other endpoint, i.e. when $k=1,(1.11)$ is an equality for $\mathbb{1}_{K}$, evident from expressing the integral in spherical coordinates.

For the affine Grassmannian $M_{n, k}$, an inequality of Schneider [41] states that if $K$ is a convex body in $\mathbb{R}^{n}$ and $1 \leqslant k \leqslant n-1$, then

$$
\int_{M_{n, k}}|K \cap F|^{n+1} d v_{n, k}(F) \leqslant \frac{\omega_{k}^{n+1} \omega_{n(k+1)}}{\omega_{n}^{k+1} \omega_{k(n+1)}}|K|^{k+1} ;
$$

when $k>1$, equality holds if and only if $K$ is an $n$-dimensional ellipsoid; when $k=1$, equality holds if and only if $K$ is a convex body, which follows from the classical Crofton formula (e.g., [42, Theorem 5.1.1]).

While many of the latter inequalities also hold for non-convex sets, the equality cases require additional care. Gardner [17] generalized (1.11) and (1.12), among other related inequalities, to the class of bounded, Borel measurable sets with a precise characterization of equality cases, making use of results due to Pfiefer [36], [37]. In this paper, we extend such inequalities to bounded integrable functions. The analysis of equality cases in the functional setting rests heavily on their results.

Theorem 1.2. Let $1 \leqslant k \leqslant n-1$ and let $f$ be a non-negative, bounded integrable function on $\mathbb{R}^{n}$. Then

$$
\int_{G_{n, k}} \frac{\left(\int_{E} f(x) d x\right)^{n}}{\left\|\left.f\right|_{E}\right\|_{\infty}^{n-k}} d \mu_{n, k}(E) \leqslant \frac{\omega_{k}^{n}}{\omega_{n}^{k}}\left(\int_{\mathbb{R}^{n}} f(x) d x\right)^{k} .
$$

We also discuss the equality cases in the latter theorem under a mild assumption on $f$ in which case equality holds in (1.13) when $k>1$ if and only if $f=a \mathbb{1}_{\mathcal{E}}$ a.e., where $\mathcal{E}$ is an origin-symmetric ellipsoid and $a$ is a positive constant. Furthermore, we prove a more genaral statement for $q \leqslant k$ different functions, as well as different powers (see Section 5).

The corresponding result on $M_{n, k}$ is the following inequality. 
Theorem 1.3. Let $1 \leqslant k \leqslant n-1$ and let $f$ be non-negative, bounded integrable function on $\mathbb{R}^{n}$. Then

$$
\int_{M_{n, k}} \frac{\left(\int_{F} f(x) d x\right)^{n+1}}{\left\|\left.f\right|_{F}\right\|_{\infty}^{n-k}} d v_{n, k}(F) \leqslant \frac{\omega_{k}^{n+1} \omega_{n(k+1)}}{\omega_{n}^{k+1} \omega_{k(n+1)}}\left(\int_{\mathbb{R}^{n}} f(x) d x\right)^{k+1} .
$$

Under a mild assumption on $f$, we also prove that equality holds in (1.14) when $k>1$ if and only if $f=a \mathbb{1}_{\mathcal{E}}$ a.e., where $\mathcal{E}$ is an ellipsoid and $a$ is a positive constant.

One can interpret Theorem 1.3 as an inequality about the $k$-plane transform. Recall that the $k$-plane transform $T_{n, k}$ applied to a function $f$ on $\mathbb{R}^{n}$ is defined by

$$
T_{n, k}(f)(F)=\int_{F} f(x) d x \quad\left(F \in M_{n, k}\right) .
$$

When $k=n-1, T_{n, k}$ is the Radon transform and when $k=1$, it is the Xray transform. The $k$-plane transform satisfies several key inequalites. In particular, for each $q \in[1, n+1]$, there is a unique $p \in[1,(n+1) /(k+1)]$ such that

$$
\left\|T_{n, k}(f)\right\|_{q} \leqslant C(n, k, q)\|f\|_{p}
$$

for all $f \in L_{p}$. The latter is a special case of a result due to Christ [10], extending work by Drury [13]; see also the article of Baernstein and Loss [1] for related work and a conjecture about the extremal functions; for recent research in this direction, see Christ [9], Druout [12] and Flock [15] and the references therein. The endpoint inequality $q=n+1$ and $p=(n+1) /(k+1)$ in $(1.16)$ also satisfies an affine-invariance property [9], [12].

Organization: We close the introduction with a few words on the main tools that we use and the organization of the paper. Section 2 is reserved for notation and background results, including formulas from integral geometry such as the Blaschke-Petkantschin formulas. In Section 3, we treat affine invariance using the structure of semi-simple Lie groups which we then specialize to the Grassmannian and affine Grassmannian. In Section 4, we recall a functional version of Busemann's random simplex inequality [7], and its variant due to Groemer [19], [20], from [33]; the latter makes essential use of Christ's form [10] of the Rogers-Brascamp-Lieb-Luttinger inequality [38], [5]. The ratios in (1.8) and (1.9) arise naturally in a suitable normalized form of the main 
inequality in [33]. In Section 5, we prove Theorems 1.2 and 1.3. We finish the paper in Section 6 with a more general version of Theorem 1.1 and we discuss connections to the Hyperplane Conjecture from convex geometry.

\section{Preliminaries}

The setting is $\mathbb{R}^{n}$ with the canonical inner-product $\langle\cdot, \cdot\rangle$, Euclidean norm $|\cdot|$ and standard unit vector basis $e_{1}, \ldots, e_{n}$. We also use $|\cdot|$ for Lebesgue measure and the absolute value of a scalar, the use of which will be clear from the context. The Euclidean ball of radius one is $B_{2}^{n}$ with volume $\omega_{n}=\left|B_{2}^{n}\right|$. We reserve $D_{n}$ for the Euclidean ball of volume one, i.e., $D_{n}=r_{n} B_{2}^{n}$, where $r_{n}=\omega_{n}^{-1 / n}$. The unit sphere is $S^{n-1}$ and is equipped with the Haar probability measure $\sigma$. As mentioned, the Haar probability measure on the Grassmannian $G_{n, k}$ is denoted by $\mu_{n, k}$. The affine Grassmannian $M_{n, k}$ is equipped with a measure as follows: for $A \subset M_{n, k}$,

$$
v_{n, k}(A)=\int_{G_{n, k}}\left|\left\{x \in E^{\perp}: x+E \in A\right\}\right| d \mu_{n, k}(E) .
$$

Henceforth, we will write simply $d F$ rather than $d v_{n, k}(F)$ for integrals over $M_{n, k}$; similarly, $d E$ instead of $d \mu_{n, k}(E)$ for integrals on $G_{n, k}$. Note that $\mu_{n, k}$ is a probability measure while $v_{n, k}$ is normalized so that $v_{n, k}(\{F \in$ $\left.\left.M_{n, k}: F \cap B_{2}^{n} \neq \emptyset\right\}\right)=\omega_{n-k}$. We use $c_{1}, c_{2}, C, \ldots$ etc for positive numeric constants.

We will make use of the following integral geometric identities, often referred to as the Blaschke-Petkantschin formulas; see e.g., [42, Chapter 7.2], [13],[17, Lemmas 5.1 \& 5.5], [28]; see also the generalization given in $[30$, Appendix A].

Theorem 2.1. Let $1 \leqslant q \leqslant k \leqslant n$. Suppose that $G$ is a non-negative, Borel measurable function on $\left(\mathbb{R}^{n}\right)^{q}$. Then

$$
\begin{array}{rl}
\int_{\left(\mathbb{R}^{n}\right)^{q}} & G\left(x_{1}, \ldots, x_{q}\right) d x_{1} \ldots d x_{q} \\
= & c_{n, k, q} \int_{G_{n, k}} \int_{E^{q}} G\left(x_{1}, \ldots, x_{q}\right)\left|\operatorname{conv}\left\{0, x_{1}, \ldots, x_{q}\right\}\right|^{n-k} d x_{1} \ldots d x_{q} d E,
\end{array}
$$

where

$$
c_{n, k, q}=(q !)^{n-k} \frac{\omega_{n-q+1} \cdots \omega_{n}}{\omega_{k-q+1} \cdots \omega_{k}} .
$$


Theorem 2.2. Let $1 \leqslant q \leqslant k \leqslant n$. Suppose that $G$ is a non-negative Borel function on $\left(\mathbb{R}^{n}\right)^{q+1}$. Then

$$
\begin{aligned}
& \int_{\left(\mathbb{R}^{n}\right)^{q+1}} G\left(x_{1}, \ldots, x_{q+1}\right) d x_{1} \ldots d x_{q+1} \\
& =c_{n, k, q} \int_{M_{n, k}} \int_{F^{q+1}} G\left(x_{1}, \ldots, x_{q+1}\right)\left|\operatorname{conv}\left\{x_{1}, \ldots, x_{q+1}\right\}\right|^{n-k} d x_{1} \ldots d x_{q+1} d F,
\end{aligned}
$$

where $c_{n, k, q}$ is defined in (2.2).

If $A \subset \mathbb{R}^{n}$ is a Borel set with finite volume, the symmetric rearrangement $A^{*}$ of $A$ is the (open) Euclidean ball centered at the origin whose volume is equal to that of $A$. The symmetric decreasing rearrangement of $1_{A}$ is defined by $\left(1_{A}\right)^{*}:=1_{A^{*}}$. If $f: \mathbb{R}^{n} \rightarrow \mathbb{R}^{+}$is an integrable function, its symmetric decreasing rearrangement $f^{*}$ is defined by

$$
f^{*}(x)=\int_{0}^{\infty} 1_{\{f>t\}}^{*}(x) d t=\int_{0}^{\infty} 1_{\{f>t\}^{*}}(x) d t .
$$

The latter can be compared with the "layer-cake representation" of $f$ :

$$
f(x)=\int_{0}^{\infty} 1_{\{f>t\}}(x) d t
$$

see [25, Theorem 1.13]. The function $f^{*}$ is radially-symmetric, decreasing and equimeasurable with $f$, i.e., $\{f>\alpha\}$ and $\left\{f^{*}>\alpha\right\}$ have the same volume for each $\alpha>0$. By equimeasurability one has $\|f\|_{p}=\left\|f^{*}\right\|_{p}$ for each $1 \leqslant p \leqslant \infty$, where $\|\cdot\|_{p}$ denotes the $L_{p}\left(\mathbb{R}^{n}\right)$-norm. We refer the reader to the book [25] for further background material on rearrangements of functions.

\section{Affine invariance}

In this section we discuss linear and affine invariance properties of the quantities in (1.8) and (1.9), respectively, as well as generalizations. We start with the former and prove the following theorem.

Theorem 3.1. Let $m$ be a positive integer and let $p_{i}, \alpha_{i}$, for $i=1, \ldots, m$ be real numbers. Let $f_{i}$ be bounded functions on $\mathbb{R}^{n}, f_{i} \in L^{p_{i}}\left(\mathbb{R}^{n}\right)$. Define

$$
I\left(f_{1}, \ldots, f_{m}\right):=\int_{G_{n, k}} \prod_{i=1}^{m}\left\|\left.f_{i}\right|_{x}\right\|_{p_{i}}^{\alpha_{i}} d x .
$$


Whenever this quantity is finite and $\sum_{i=1}^{m} \frac{\alpha_{i}}{p_{i}}=n$, for any volume-preserving linear transformation $g$, we have

$$
I\left(g \cdot f_{1}, \ldots, g \cdot f_{m}\right)=I\left(f_{1}, \ldots, f_{m}\right),
$$

where $g \cdot f_{i}(t)=f_{i}\left(g^{-1} t\right)$.

Remark 3.2. Letting $p_{i}, \alpha_{i}, q_{i}, \beta_{i}$ satisfy $\sum_{i=1}^{m}\left(\frac{\alpha_{i}}{p_{i}}-\frac{\beta_{j}}{q_{j}}\right)=n$, Theorem 3.1 yields the linear invariance of

$$
\int \prod_{G_{n, k}}^{m} \frac{\left\|\left.f_{i}\right|_{x}\right\|_{p_{i}}^{\alpha_{i}}}{\left\|\left.f_{i}\right|_{x}\right\|_{q_{i}}^{\beta_{i}}} d x,
$$

and letting $q_{i} \rightarrow \infty$, also of

$$
\int \prod_{G_{n, k}}^{m} \frac{\left\|\left.f_{i}\right|_{x}\right\|_{p_{i}}^{\alpha_{i}}}{\left\|\left.f_{i}\right|_{x}\right\|_{\infty}^{\beta_{i}}} d x .
$$

Note that in the latter case there are no restriction on the $\beta_{i}$ 's.

Grinberg's approach [18], which in turn draws on Furstenberg-Tzkoni [16], can be adapted to our setting, although we prefer to give a more self-contained proof using the structure of semi-simple Lie groups. For this reason, the notation in this section differs somewhat from the rest of the paper.

\subsection{Semi-simple Lie groups}

We recall some basic facts from the theory of semi-simple Lie groups as needed for our later discussion about the Grassmannian manifold. We follow the presentation from [31]. Further information and details about this topic can be found for example in [24].

Let $G$ be a non-compact connected semi-simple Lie group with a finite center. We denote its Lie algebra by $\mathfrak{g}$. An involution $\theta: G \rightarrow G$ is called a Cartan involution if $K=G^{\theta}:=\{a \in G: \theta(a)=a\}$ is a maximal compact subgroup of $G$. In this case $K$ is connected. We fix a Cartan involution $\theta$ on $G$ and the corresponding maximal compact subgroup $K$. The derived involution $\dot{\theta}: \mathfrak{g} \rightarrow \mathfrak{g}$ will also be denoted by $\theta$. We have $\mathfrak{g}=\mathfrak{k} \oplus \mathfrak{s}$, where $\mathfrak{k}=\mathfrak{g}^{\theta}$ is the Lie algebra of $K$ and $\mathfrak{s}=\{X \in \mathfrak{g}: \theta(X)=-X\}$. 
Moreover, $[\mathfrak{k}, \mathfrak{k}] \subset \mathfrak{k},[\mathfrak{s}, \mathfrak{s}] \subset \mathfrak{k},[\mathfrak{k}, \mathfrak{s}] \subset \mathfrak{s}$. By Ad and ad, we denote the adjoint representation of the Lie group $G$ and of the Lie algebra $\mathfrak{g}$, respectively. The Killing form on $\mathfrak{g}$ is given by $\langle X, Y\rangle:=\operatorname{Tr}(\operatorname{ad}(X) \operatorname{ad}(Y))$. And the product

$$
(X, Y):=-\langle X, \theta(Y)\rangle
$$

is an inner product on $\mathfrak{g}$. Note that $\operatorname{ad}(X)^{*}=-\operatorname{ad}(\theta(X))$. In particular, for $X \in \mathfrak{s}, \operatorname{ad}(X)$ is a symmetric operator and hence diagonalizable over the reals. Let $\mathfrak{a} \subset \mathfrak{s}$ be abelian. Then ad $\mathfrak{a}$ is a family of commuting symmetric transformations and thus can be diagonalized simultaneously, with real eigenvalues. For each linear functional $\lambda$ on $\mathfrak{a}, \lambda \in \mathfrak{a}^{*}$, let

$$
\mathfrak{g}_{\lambda}=\{X \in \mathfrak{g}:[H, X]=\lambda(H) X \text { for all } H \in \mathfrak{a}\}
$$

and set

$$
\mathfrak{m}=\{X \in \mathfrak{g}:[H, X]=0 \text { for all } H \in \mathfrak{a} \text { and } X \perp \mathfrak{a}\} .
$$

Then $\mathfrak{g}_{0}=\mathfrak{m} \oplus \mathfrak{a}$. Let $\Delta=\left\{\lambda \in \mathfrak{a}^{*} \backslash\{0\}: \mathfrak{g}_{\lambda} \neq\{0\}\right\}$. Elements in $\Delta$ are called restricted roots. We have

$$
\mathfrak{g}=\mathfrak{m} \oplus \mathfrak{a} \oplus \bigoplus_{\lambda \in \Delta} \mathfrak{g}_{\lambda}
$$

For $\lambda, \mu \in \Delta \cup\{0\}$, we have $\left[\mathfrak{g}_{\lambda}, \mathfrak{g}_{\mu}\right] \subset \mathfrak{g}_{\lambda+\mu}$. In particular, [m $\left.\oplus \mathfrak{a}, \mathfrak{g}_{\lambda}\right] \subset \mathfrak{g}_{\lambda}$ for all $\lambda \in \Delta$. Further, let $\mathfrak{a}_{\mathfrak{r}}=\{H \in \mathfrak{a}: \lambda(H) \neq 0$ for all $\lambda \in \Delta\}$. Fix $H \in \mathfrak{a}_{\mathrm{r}}$ and let $\Delta^{+}=\{\lambda \in \Delta: \lambda(H)>0\}$. Elements in $\Delta^{+}$are called positive roots. We have $\Delta=\Delta^{+} \cup-\Delta^{+}, \Delta^{+} \cap-\Delta^{+}=\emptyset$ and $\left(\Delta^{+}+\Delta^{+}\right) \cap \Delta \subset \Delta^{+}$. It follows that

$$
\mathfrak{n}:=\bigoplus_{\lambda \in \Delta^{+}} \mathfrak{g}_{\lambda}
$$

is a nilpotent subalgebra of $\mathfrak{g}$ normalized by the Lie algebra $\mathfrak{p}:=\mathfrak{m} \oplus \mathfrak{a} \oplus \mathfrak{n}$. In fact, $\mathfrak{p}$ is a parabolic subalgebra of $\mathfrak{g}$. It is maximal if $\operatorname{dim} \mathfrak{a}=1$.

Let $P:=\{g \in G: \operatorname{Ad}(g) \mathfrak{p} \subset \mathfrak{p}\}$. Then $P$ is a closed subgroup of $G$ with the Lie algebra $\mathfrak{p}$. Let $A:=\exp \mathfrak{a}$ and $N:=\exp n$ be analytic subgroups of $G$ with Lie algebras $\mathfrak{a}$ and $\mathfrak{n}$, respectively. The groups $A, N$ are closed. $A$ is abelian and $N$ is nilpotent. Denote by $M_{o}$ the analytic subgroup of $G$ with the Lie algebra m. Let $M:=Z_{K}(A) M_{o}$, where $Z_{K}(A)$ stands for the centralizer of $A$ in $K . M$ is a closed subgroup of $G$ with finitely many connected components. The map $M \times A \times N \ni(m, a, n) \mapsto \operatorname{man} \in P$ is an analytic diffeomorphism. We have $G=K P$. Further, let $L:=K \cap M$ and $\widetilde{X}:=K / L$, then

$$
K \cap P=L \text { and } \widetilde{X}=G / P
$$


The group $G$ acts on $\widetilde{X}$. Write

$$
G \ni g=k(g) m(g) a(g) n(g) \quad((k(g), m(g), a(g), n(g)) \in K \times M \times A \times N) .
$$

The map $g \mapsto(a(g), n(g))$ is analytic and the map $g \mapsto a(g)$ is right $M N$ invariant. Thus we can view $a(\cdot)$ as a map $G / M N \rightarrow A$. The elements $k(g), m(g)$ are not uniquely defined. However, the map

$$
g \mapsto k(g) L \in \widetilde{X}
$$

is well-defined and analytic. Set $x_{0}:=e L$, where $e$ denotes the identity element of $G$. The action of $G$ on $\widetilde{X}$ can now be described by $g \cdot\left(k \cdot x_{o}\right)=$ $k(g k) \cdot x_{o}$. For $x=k \cdot x_{o} \in \widetilde{X}$ and $g \in G$, we set $a(g x):=a(g k)$.

We normalize the invariant measure on $\widetilde{X}$ to have total mass one. For $f \in C(\widetilde{X})$,

$$
\int_{\widetilde{X}} f(x) d x=\int_{K} f\left(k \cdot x_{0}\right) d k .
$$

For $\lambda \in \mathfrak{a}^{*}$ define a homomorphism $\chi_{\lambda}: P \rightarrow \mathbb{R}$ by

$$
\chi_{\lambda}(m \exp (H) n)=e^{\lambda(H)} \text {, where } m \in M, H \in \mathfrak{a}, n \in N .
$$

The shorthand notation for $\chi_{\lambda}(p)$ is $p^{\lambda}$. For $\lambda \in \Delta$, set $m_{\lambda}:=\operatorname{dim} \mathfrak{g}_{\lambda}$ and $\rho:=\frac{1}{2} \sum_{\lambda \in \Delta^{+}} m_{\lambda} \lambda \in \mathfrak{a}^{*}$. Note that $p^{2 \rho}=|\operatorname{det} \operatorname{Ad}(p)|_{\mathfrak{n}} \mid$.

We shall need the following well known lemma.

Lemma 3.3. Let $f \in L^{1}(\widetilde{X})$ and $g \in G$. Then

$$
\int_{\widetilde{X}} f(g \cdot x) a(g x)^{-2 \rho} d x=\int_{\widetilde{X}} f(x) d x .
$$

Proof. See Lemma 5.19 on p. 197 in [21]. This result is formulated there in a slightly different form. However, the precise equality appears in the proof as equation (25).

\subsection{The Grassmannian manifold}

Now we apply the general structure theory of semi-simple Lie groups discussed above to the special case of Grassmann manifolds.

Let $G_{n, k}$ denote the Grassmann manifold of all oriented $k$-dimensional subspaces of $\mathbb{R}^{n}$ and set $r=n-k$. Note that $G_{n, k} \cong G_{n, r}$.

Set $G=\operatorname{SL}(n)$, then $\mathfrak{g}$ is the set of $n \times n$ matrices with trace zero. The homomorphism $\theta: G \rightarrow G: x \rightarrow x^{-t r}$ is a Cartan involution on $G$ with 
$K=G^{\theta}=\mathrm{SO}(n)$. The corresponding Cartan involution on $\mathfrak{g}$ is $\theta(X)=$ $-X^{t r}$. Denote by $\mathrm{M}(n)$ the set of $n \times n$ matrices. We have

$$
\begin{aligned}
& \mathfrak{k}=\left\{X \in M(n): X^{t r}=-X \text { and } \operatorname{Tr}(X)=0\right\}, \\
& \mathfrak{s}=\left\{X \in M(n): X^{t r}=X \text { and } \operatorname{Tr}(X)=0\right\} .
\end{aligned}
$$

The Killing form on $\mathfrak{g}$ is given by $\langle X, Y\rangle=2 n \operatorname{Tr}(X Y)$. For $l \in \mathbb{N}$, denote by $\mathrm{I}_{l}$ the $l \times l$ identity matrix. Let

$$
H_{o}=\left(\begin{array}{cc}
\frac{r}{n} \mathrm{I}_{k} & 0 \\
0 & -\frac{k}{n} \mathrm{I}_{r}
\end{array}\right) \in \mathfrak{s} .
$$

We define $\mathfrak{a}:=\mathbb{R} H_{o}$, then $\mathfrak{m}=\left\{X \in z_{\mathfrak{g}}(\mathfrak{a}):\left\langle X, H_{o}\right\rangle=0\right\}$. Fix $\alpha \in \mathfrak{a}^{*}$ so that $\alpha\left(H_{o}\right)=1$. We choose $\Delta=\{\alpha,-\alpha\}$ and $\Delta^{+}=\{\alpha\}$. We have

$$
\begin{aligned}
& \mathfrak{m} \oplus \mathfrak{a}=\left\{\left(\begin{array}{cc}
X & 0 \\
0 & Y
\end{array}\right): \begin{array}{c}
X \in M(k), \\
Y \in M(r)
\end{array} \text { and } \operatorname{Tr} X+\operatorname{Tr} Y=0\right\} \\
& =\mathfrak{s}(\mathfrak{g l}(k) \times \mathfrak{g l}(r)), \\
& \mathfrak{n}=\left\{\left(\begin{array}{cc}
0 & X \\
0 & 0
\end{array}\right): X \in M(k \times r)\right\}, \\
& M A=\left\{m(a, b):=\left(\begin{array}{ll}
a & 0 \\
0 & b
\end{array}\right): \begin{array}{l}
a \in \mathrm{GL}(k), \\
b \in \mathrm{GL}(r)
\end{array} \text { and } \operatorname{det} a \operatorname{det} b=1\right\} \\
& =\mathrm{S}(\mathrm{GL}(k) \times \mathrm{GL}(r)) \text {, } \\
& A=\left\{\left(\begin{array}{cc}
s \mathrm{I}_{k} & 0 \\
0 & t \mathrm{I}_{r}
\end{array}\right): s, t>0 \text { with } s^{k} t^{r}=1\right\}, \\
& M=\left\{\left(\begin{array}{ll}
a & 0 \\
0 & b
\end{array}\right): \operatorname{det} a, \operatorname{det} b= \pm 1 \text { and } \operatorname{det} a \operatorname{det} b=1\right\}, \\
& N=\left\{n(X):=\left(\begin{array}{cc}
\mathrm{I}_{k} & X \\
0 & \mathrm{I}_{r}
\end{array}\right): X \in M(k \times r)\right\}, \\
& P=\left\{\left(\begin{array}{cc}
a & X \\
0 & b
\end{array}\right): \begin{array}{ll}
a \in \mathrm{GL}(k), & \operatorname{det} a \operatorname{det} b=1, \\
b \in \mathrm{GL}(r), & X \in M(k \times r)
\end{array}\right\}, \\
& L=\mathrm{S}(\mathrm{O}(k) \times \mathrm{O}(r)) \text {. }
\end{aligned}
$$

Let $e_{1}, \ldots, e_{n}$ be the canonical basis for $\mathbb{R}^{n}$. Set $x_{o}=\mathbb{R} e_{1} \oplus \cdots \oplus \mathbb{R} e_{k} \in G_{n, k}$. We have $G_{n, k}=K \cdot x_{o} \cong K / L=G / P$. 
We identify $\mathfrak{a}^{*}$ with $\mathbb{R}$ via $\lambda \mapsto \frac{n}{k r} \lambda\left(H_{0}\right)$. The inverse of this map is $z \mapsto z \frac{k r}{n} \alpha$. Since $\operatorname{dim} \mathfrak{n}=k r$, we have

$$
\mathfrak{a}^{*} \ni \rho=\frac{k r}{2} \alpha \longleftrightarrow \frac{n}{2} \in \mathbb{R} .
$$

For $z \in \mathbb{R}$, we write $p^{z}$ instead of $p^{z \frac{k r}{n} \alpha}$.

\subsection{Linear invariance for functions on $G_{n, k}$}

For $g \in \mathrm{SL}(n)$ and $x \in G_{n, k}$ denote by $J_{g}(x)$ the Jacobian determinant of the transformation $x \mapsto g x$. Then for $f \in L^{1}(x)$,

$$
\int_{x} f(t)\left|J_{g}(x)\right| d t=\int_{g x} f\left(g^{-1} t\right) d t .
$$

Lemma 3.4. For $k \in \mathrm{SO}(n)$ and $x \in G_{n, k}$, we have $\left|J_{k}(x)\right|=1$.

Proof. Let $K \subset \mathbb{R}^{n}$ be measurable and $f=\mathbb{1}_{K}$ be the characteristic function of $K$. For $g \in \operatorname{SL}(n)$, we compute

$$
\int_{g x} \mathbb{1}_{K}\left(g^{-1} t\right) d t=\int_{g x} \mathbb{1}_{g K}(t) d t=|g K \cap g x|=|g(K \cap x)| .
$$

If $g \in \mathrm{SO}(n)$, then $|g(K \cap x)|=|(K \cap x)|=\int_{x} \mathbb{1}_{K}(t) d t$. And the claim follows for characteristic functions.

By an analogous computation, the claim follows for simple functions. For a general function $f$, the claim follows by approximating $f$ from below by simple functions.

Recall the following multiplicative property of the Jacobian: Let $T$ : $Z \rightarrow Y$ and $S: Y \rightarrow X$, then $S \circ T: Z \rightarrow X$ and

$$
J_{S \circ T}(z)=J_{S}(T(z)) J_{T}(z) \text { with } z \in Z \text {. }
$$

Lemma 3.5. For $z \in \mathbb{R}, x \in G_{n, k}$ and $g \in \mathrm{SL}(n)$, we have $\left|J_{g}(x)\right|^{z}=a(g x)^{z}$.

Proof. Write $g=k p$ with $k \in K$ and $p \in P$. By the multiplicative property of the Jacobian, we have

$$
\left|J_{g}\left(x_{o}\right)\right|=\left|J_{k p}\left(x_{o}\right)\right|=\left|J_{k}\left(p \cdot x_{o}\right) J_{p}\left(x_{o}\right)\right|=\left|J_{p}\left(x_{o}\right)\right|,
$$

where the last equality follows by Lemma 3.4. 
Let $y \in \mathbb{R}^{k}$, then $\tilde{y}=(y, 0, \ldots, 0) \in x_{0}$. Decompose $p=m(a, b) n(X)$ and compute

$$
p \cdot \tilde{y}=\left(\begin{array}{ll}
a & 0 \\
0 & b
\end{array}\right)\left(\begin{array}{cc}
\mathrm{I}_{k} & X \\
0 & \mathrm{I}_{r}
\end{array}\right)\left(\begin{array}{l}
y \\
0
\end{array}\right)=\left(\begin{array}{cc}
a & X \\
0 & b
\end{array}\right)\left(\begin{array}{l}
y \\
0
\end{array}\right)=\left(\begin{array}{c}
a y \\
0
\end{array}\right) .
$$

Thus $J_{p}\left(x_{o}\right)=\operatorname{det} a$.

For some $m_{o} \in M$ and $s \in \mathbb{R}, m(a, b)=m_{o} \exp \left(s H_{o}\right)$. Let $m_{o}=\left(\begin{array}{cc}u & 0 \\ 0 & v\end{array}\right)$ and note that $\exp \left(s H_{o}\right)=\left(\begin{array}{cc}e^{s \frac{r}{n}} I_{k} & 0 \\ 0 & e^{-s \frac{k}{n}} \mathrm{I}_{r}\end{array}\right)$. It follows that $a=e^{s \frac{r}{n}} u$. Hence $\operatorname{det} a=e^{s \frac{k r}{n}} \operatorname{det} u$ and so $|\operatorname{det} a|=e^{s \frac{k r}{n}}$.

Next observe: $\exp \left(s H_{o}\right)^{z}=\chi_{z \frac{k r}{n} \alpha}\left(\exp \left(s H_{0}\right)\right)=e^{z \frac{k r}{n} \alpha\left(s H_{0}\right)}=e^{s \frac{k r}{n} z}$. Thus we have shown

$$
\left|J_{g}\left(x_{o}\right)\right|^{z}=\left|J_{p}\left(x_{o}\right)\right|^{z}=|\operatorname{det} a|^{z}=e^{s \frac{k r}{n} z}=\exp \left(s H_{o}\right)^{z}=a(g)^{z} .
$$

Write a general $x \in G_{n, k}$ as $x=k \cdot x_{0}$. We have $\left|J_{g}(x)\right|=\left|J_{g}\left(k \cdot x_{o}\right)\right|=\mid J_{g}(k$. $\left.x_{o}\right) J_{k}\left(x_{o}\right)|=| J_{g k}\left(x_{o}\right) \mid$. Hence

$$
\left|J_{g}(x)\right|^{z}=\left|J_{g k}\left(x_{o}\right)\right|^{z}=a(g k)^{z}=a(g x)^{z} .
$$

Substituting the result of Lemma 3.5 into (3.2) yields

$$
a(g x) \int_{x} f(t) d t=\int_{g x} f\left(g^{-1} t\right) d t .
$$

Proof of Theorem 3.1. Fix $m \in \mathbb{N}$ and let $f_{i}, p_{i}, \alpha_{i}, g$ be as described in the statement of the theorem. We compute

$$
\begin{aligned}
I\left(g \cdot f_{1}, \ldots, g \cdot f_{m}\right) & =\int_{G_{n, k}} \prod_{i=1}^{m}\left(\int_{x}\left|f_{i}\left(g^{-1} t\right)\right|^{p_{i}} d t\right)^{\frac{\alpha_{i}}{p_{i}}} d x \\
& =\int_{G_{n, k}} a(g x)^{-n} \prod_{i=1}^{m}\left(\int_{g x}\left|f_{i}\left(g^{-1} t\right)\right|^{p_{i}} d t\right)^{\frac{\alpha_{i}}{p_{i}}} d x \\
& =\int_{G_{n, k}} a(g x)^{-n} \prod_{i=1}^{m}\left(a(g x) \int_{x}\left|f_{i}(t)\right|^{p_{i}} d t\right)^{\frac{\alpha_{i}}{p_{i}}} d x
\end{aligned}
$$




$$
\begin{aligned}
& =\int_{G_{n, k}} a(g x)^{-n} a(g x)^{\sum \frac{\alpha_{i}}{p_{i}}} \prod_{i=1}^{m}\left\|\left.f_{i}\right|_{x}\right\|_{p_{i}}^{\alpha_{i}} d x \\
& =I\left(f_{1}, \ldots, f_{m}\right) .
\end{aligned}
$$

The second equality follows by Lemma 3.3 and (3.1). The third equality follows by (3.3).

\subsection{Affine invariance for functions on $M_{n, k}$}

The linear-invariance property from the previous section can be transferred to an affine-invariance property on the affine Grassmannian.

Theorem 3.6. Let $m$ be a positive integer and let $p_{i}, \alpha_{i}$, for $i=1, \ldots, m$ be real numbers. Let $f_{i}$ be bounded functions on $\mathbb{R}^{n}, f_{i} \in L^{p_{i}}\left(\mathbb{R}^{n}\right)$. Define

$$
\tilde{I}\left(f_{1}, \ldots, f_{m}\right):=\int_{M_{n, k}} \prod_{i=1}^{m}\left\|\left.f_{i}\right|_{x}\right\|_{p_{i}}^{\alpha_{i}} d x .
$$

Whenever this quantity is finite and $\sum_{i=1}^{m} \frac{\alpha_{i}}{p_{i}}=n+1$, for any volume-preserving affine transformation $g$, we have

$$
\tilde{I}\left(g \cdot f_{1}, \ldots, g \cdot f_{m}\right)=\tilde{I}\left(f_{1}, \ldots, f_{m}\right),
$$

where $g \cdot f_{i}(t)=f_{i}\left(g^{-1} t\right)$.

To prove this theorem we will need an analog of Lemma 3.3, for which, in turn, we need a couple of simple observations.

Lemma 3.7. For $x \in G_{n, k}$ and $g \in \operatorname{SL}(n)$, we have $\left|J_{g}\left(x^{\perp}\right)\right|=\left|J_{g}(x)\right|^{-1}$. Proof.

$$
\begin{aligned}
\int_{\mathbb{R}^{n}} g \cdot f(z) d z & =\int_{\mathbb{R}^{n}} f(z) d z=\int_{x^{\perp}} \int_{x} f(t+s) d t d s \\
& =\int_{x^{\perp}} \int_{g x} f\left(g^{-1} t+s\right)\left|J_{g}(x)\right|^{-1} d t d s \\
& =\int_{g x^{\perp}} \int_{g x} f\left(g^{-1} t+g^{-1} s\right)\left|J_{g}(x)\right|^{-1} d t\left|J_{g}\left(x^{\perp}\right)\right|^{-1} d s \\
& =\left|J_{g}(x)\right|^{-1}\left|J_{g}\left(x^{\perp}\right)\right|^{-1} \int_{\mathbb{R}^{n}} g \cdot f(z) d z,
\end{aligned}
$$

where we applied (3.2) twice. 
Lemma 3.8. Let $g \in \operatorname{SL}(n)$. For $y \in M_{n, k}$, let $x \in G_{n, k}$ and $s \in \mathbb{R}^{n}$ be so that $y=x+$ s. Then $\left|J_{g}(y)\right|=\left|J_{g}(x)\right|$.

Proof.

$$
\begin{aligned}
\int_{g y} f\left(g^{-1} z\right)\left|J_{g}(y)\right|^{-1} d z & =\int_{x+s} f(z) d z=\int_{x} f(t+s) d t \\
& =\int_{g x} f\left(g^{-1} t+s\right)\left|J_{g}(x)\right|^{-1} d t \\
& =\int_{g(x+s)} f\left(g^{-1} z\right)\left|J_{g}(x)\right|^{-1} d z \\
& =\int_{g y} f\left(g^{-1} z\right)\left|J_{g}(x)\right|^{-1} d z,
\end{aligned}
$$

where we again employed (3.2).

Lemma 3.9. Let $g \in \operatorname{SL}(n)$ and $f \in L^{1}\left(M_{n, k}\right)$. Then

$$
\int_{M_{n, k}} f(g y)\left|J_{g}(y)\right|^{-(n+1)} d y=\int_{M_{n, k}} f(y) d y .
$$

Proof.

$$
\begin{aligned}
\int_{M_{n, k}} f(g y) d y & =\int_{G_{n, k}} \int_{x^{\perp}} f(g(x+s)) d s d x \\
& =\int_{G_{n, k}} \int_{x^{\perp}} f(x+g s) d s\left|J_{g^{-1}}(x)\right|^{-n} d x \\
& =\int_{G_{n, k}} \int_{g x^{\perp}} f(x+s)\left|J_{g}\left(x^{\perp}\right)\right|^{-1} d s\left|J_{g}(x)\right|^{n} d x \\
& =\int_{G_{n, k}} \int_{g x^{\perp}} f(x+s) d s\left|J_{g}(x)\right|^{n+1} d x \\
& =\int_{M_{n, k}} f(y)\left|J_{g}(y)\right|^{n+1} d y,
\end{aligned}
$$

where the second equality follows by Lemma 3.3 along with Lemma 3.5.

Proof of Theorem 3.6. The result follows by an analogous computation to the one in the proof of Theorem 3.1.

For related affine invariance properties, see [9] and [12]. 


\section{Functional forms of isoperimetric inequalities}

We start by recalling the main result from [33]. For positive integers $k, n$ and vectors $x_{1}, \ldots, x_{k}$ in $\mathbb{R}^{n}$, we view the $k \times n$ matrix $\left[x_{1} \cdots x_{k}\right]$ as an operator from $\mathbb{R}^{k}$ to $\mathbb{R}^{n}$. If $C \subset \mathbb{R}^{k}$, then

$$
\left[x_{1} \cdots x_{k}\right] C=\left\{\sum_{i=1}^{k} c_{i} x_{i}: c=\left(c_{i}\right) \in C\right\} .
$$

For example, if $C=\operatorname{conv}\left\{0, e_{1}, \ldots, e_{k}\right\}$, then

$$
\left[x_{1} \cdots x_{k}\right] \operatorname{conv}\left\{0, e_{1}, \ldots, e_{k}\right\}=\operatorname{conv}\left\{0, x_{1}, \ldots, x_{k}\right\} .
$$

Similarly, if $x_{1}, \ldots, x_{k+1} \in \mathbb{R}^{n}$ and we consider $C=\operatorname{conv}\left\{e_{1}, \ldots, e_{k+1}\right\} \subset$ $\mathbb{R}^{k+1}$, we have

$$
\left[x_{1} \cdots x_{k+1}\right] \operatorname{conv}\left\{e_{1}, \ldots, e_{k+1}\right\}=\operatorname{conv}\left\{x_{1}, \ldots, x_{k+1}\right\} .
$$

If $\operatorname{dim} C$ denotes the dimension of the affine hull of $C$, then

$$
\operatorname{dim}\left(\left[x_{1} \cdots x_{k}\right] C\right)=\min \left(\operatorname{rank}\left(\left[x_{1} \cdots x_{k}\right]\right), \operatorname{dim} C\right) ;
$$

moreover, for almost every $x_{1}, \ldots, x_{k} \in \mathbb{R}^{n}$, we have $\operatorname{rank}\left(\left[x_{1} \cdots x_{k}\right]\right)=$ $\min (k, n)$.

Let $f_{1}, \ldots, f_{k}$ be non-negative bounded, integrable functions on $\mathbb{R}^{n}$ such that $\left\|f_{i}\right\|_{1}>0$ for each $i=1, \ldots, k$. For a compact, convex set $C \subset \mathbb{R}^{k}$ and $p \neq 0$, set

$$
\begin{aligned}
& \mathcal{F}_{C, p}\left(f_{1}, \ldots, f_{k}\right) \\
& \quad=\left(\int_{\mathbb{R}^{n}} \cdots \int_{\mathbb{R}^{n}}\left|\left[x_{1} \cdots x_{k}\right] C\right|^{p} \prod_{i=1}^{k} \frac{f_{i}\left(x_{i}\right)}{\left\|f_{i}\right\|_{1}} d x_{1} \ldots d x_{k}\right)^{1 / p} .
\end{aligned}
$$

Here $|\cdot|$ denotes $m$-dimensional Lebesgue measure, where $m=\min (k, n, \operatorname{dim} C)$.

The main result from [33] (see Theorem 3.10 and Section 4.1) is the following theorem.

Theorem 4.1. Let $k$ and $n$ be positive integers and $C \subset \mathbb{R}^{k}$ a compact convex set. Let $f_{1}, \ldots, f_{k}$ be non-negative integrable functions such that $\left\|f_{i}\right\|_{1}>0$ for $i=1, \ldots, k$. Then for each $p \neq 0$,

$$
\mathcal{F}_{C, p}\left(f_{1}, \ldots, f_{k}\right) \geqslant \mathcal{F}_{C, p}\left(f_{1}^{*}, \ldots, f_{k}^{*}\right) .
$$

Moreover, if $\left\|f_{i}\right\|_{\infty} \leqslant 1=\left\|f_{i}\right\|_{1}$ for each $i=1, \ldots, k$ and $p \geqslant 1$, then

$$
\mathcal{F}_{C, p}\left(f_{1}^{*}, \ldots, f_{k}^{*}\right) \geqslant \mathcal{F}_{C, p}\left(\mathbb{1}_{D_{n}}, \ldots, \mathbb{1}_{D_{n}}\right) .
$$


Under suitable assumptions on $C$, the condition $p \geqslant 1$ can be relaxed (e.g., when $\left|\left[x_{1} \cdots x_{k}\right] C\right|^{p}$ is coordinate-wise increasing analogous to [11, Lemma 4.3]).

In [33], the latter result was stated with the additional assumption that $k \geqslant n$ and that $C \subset \mathbb{R}^{k}$ is a convex body (so $\operatorname{dim} C=k$ ). In fact, the argument given there works for any positive integer $k$ and any compact convex set $C \subset \mathbb{R}^{k}$. If $k \leqslant n$ and $\operatorname{dim} C=k$, the matrix $X=\left[x_{1} \cdots x_{k}\right]$ represents an embedding from $\mathbb{R}^{k}$ into $\mathbb{R}^{n}$ and

$$
\left|\left[x_{1} \cdots x_{k}\right] C\right|=\operatorname{det}\left(X^{*} X\right)^{1 / 2}|C| ;
$$

see, e.g., $[14$, Chapter 3$]$. In this case, the quantities $\mathcal{F}_{C, p}^{p}\left(f_{1}, \ldots, f_{k}\right)$ are all multiples of

$$
\int_{\mathbb{R}^{n}} \cdots \int_{\mathbb{R}^{n}}\left|\operatorname{conv}\left\{0, x_{1}, \ldots, x_{k}\right\}\right|^{p} \prod_{i=1}^{k} \frac{f_{i}\left(x_{i}\right)}{\left\|f_{i}\right\|_{1}} d x_{1} \ldots d x_{k}
$$

(cf. (4.2)). When $k>n$, the geometry of $C$ plays a more significant role, and choosing $C$ suitably gives rise to a number of isoperimetric inequalities (which was our main interest in [33]).

It will be useful to have a non-normalized variant of Theorem 4.1 which relaxes the assumption $\left\|f_{i}\right\|_{\infty} \leqslant 1=\left\|f_{i}\right\|_{1}$. In fact, there are several such variants, depending on the homogeneity properties of the integrand in (4.3).

For subsequent reference, we record two basic identities concerning the volume of the sets $\left[x_{1} \cdots x_{k}\right] C$, where $x_{1}, \ldots, x_{k} \in \mathbb{R}^{n}$, and $C \subset \mathbb{R}^{k}$ is a compact convex set. Note first that for each $a>0$,

$$
\left|\left[a x_{1} \cdots a x_{k}\right] C\right|=a^{m}\left|\left[x_{1} \cdots x_{k}\right] C\right|,
$$

where $m=\min \left(\operatorname{rank}\left(\left[x_{1} \cdots x_{k}\right]\right), \operatorname{dim} C\right)$. Moreover, if $k \leqslant n$ and $\operatorname{dim} C=k$, and $a_{1}, \ldots, a_{k} \in \mathbb{R}^{+}$, then

$$
\left|\left[a_{1} x_{1} \cdots a_{k} x_{k}\right] C\right|=a_{1} \cdots a_{k}\left|\left[x_{1} \cdots x_{k}\right] C\right|,
$$

which follows from (4.6).

Corollary 4.2. Let $1 \leqslant k \leqslant n$ and $f_{1}, \ldots, f_{k}$ be non-negative, bounded integrable functions on $\mathbb{R}^{n}$ such that $\left\|f_{i}\right\|_{1}>0$ for each $i=1, \ldots, k$. For $p \in \mathbb{R}$, set

$$
\Delta_{p}^{0}\left(f_{1}, \ldots, f_{k}\right)=\int_{\mathbb{R}^{n}} \ldots \int_{\mathbb{R}^{n}}\left|\operatorname{conv}\left\{0, x_{1}, \ldots, x_{k}\right\}\right|^{p} \prod_{i=1}^{k} f_{i}\left(x_{i}\right) d x_{1} \ldots d x_{k} .
$$


Then for $p>0$,

$$
\Delta_{p}^{0}\left(f_{1}, \ldots, f_{k}\right) \geqslant\left(\prod_{i=1}^{k} \frac{\left\|f_{i}\right\|_{1}^{1+p / n}}{\omega_{n}^{1+p / n}\left\|f_{i}\right\|_{\infty}^{p / n}}\right) \Delta_{p}^{0}\left(\mathbb{1}_{B_{2}^{n}}, \ldots, \mathbb{1}_{B_{2}^{n}}\right) .
$$

When $-(n-k+1)<p<0$, the inequality is reversed. Assume additionally that $\left\{x: f_{i}(x)=\left\|f_{i}\right\|_{\infty}\right\}$ is a bounded subset of $\mathbb{R}^{n}$ for $i=1, \ldots, k$ and $p \neq 0$. Then equality holds in (4.10) for $k=n$ if and only if there is an originsymmetric ellipsoid $\mathcal{E}$ and positive constants $a_{i}, b_{i}$, such that $f_{i}=a_{i} \mathbb{1}_{b_{i} \mathcal{E}}$ a.e. for $i=1, \ldots, k$; for $k<n$, equality holds in (4.10) if and only if there are positive constants $a_{i}, b_{i}$, such that $f_{i}=a_{i} \mathbb{1}_{b_{i} B_{2}^{n}}$ a.e. for $i=1, \ldots, k$.

The condition $-(n-k+1)<p$ is needed for integrability. Since we treat the equality cases in the latter corollary but there is no discussion of equality cases in Theorem 4.1 , it will be useful to recall one step in the proof of (4.5). The basic ingredient is the next lemma, see e.g., [33, Lemma 3.5], [11, Proof of Lemma 4.3]; the equality condition is not stated in the latter articles but it is easily obtained from the proofs. Here, as above, $r_{n}=\omega_{n}^{-1 / n}$ is the radius of the Euclidean ball $D_{n}$ of volume one.

Lemma 4.3. Let $f: \mathbb{R}^{+} \rightarrow[0,1]$ and suppose that $\int_{0}^{\infty} f(r) r^{n-1} d r=\int_{0}^{r_{n}} r^{n-1} d r$. Then for any increasing function $\phi: \mathbb{R}^{+} \rightarrow \mathbb{R}^{+}$, we have

$$
\int_{0}^{\infty} \phi(r) f(r) r^{n-1} d r \geqslant \int_{0}^{r_{n}} \phi(r) r^{n-1} d r .
$$

If $\phi$ is strictly increasing, then equality holds if and only if $f=\mathbb{1}_{\left[0, r_{n}\right]}$ a.e.

Proof of Corollary 4.2. For $i=1, \ldots, k$, let $a_{i}=\left(\left\|f_{i}\right\|_{1} /\left\|f_{i}\right\|_{\infty}\right)^{1 / n}$ and let

$$
\bar{f}_{i}(x)=\frac{f_{i}\left(a_{i} x\right)}{\int_{\mathbb{R}^{n}} f_{i}\left(a_{i} y\right) d y} .
$$

Then $\left\|\bar{f}_{i}\right\|_{1}=\left\|\bar{f}_{i}\right\|_{\infty}=1$. Using homogeneity property (4.8) for the set $C=\operatorname{conv}\left\{0, e_{1}, \ldots, e_{k}\right\}$, we have

$$
\begin{aligned}
\Delta_{p}^{0}\left(f_{1}, \ldots, f_{k}\right) & =\mathcal{F}_{C, p}^{p}\left(f_{1}, \ldots, f_{k}\right) \prod_{i=1}^{k}\left\|f_{i}\right\|_{1} \\
& =\mathcal{F}_{C, p}^{p}\left(\bar{f}_{1}, \ldots, \bar{f}_{k}\right) \prod_{i=1}^{k} \frac{\left\|f_{i}\right\|^{1+p / n}}{\left\|f_{i}\right\|_{\infty}^{p / n}} .
\end{aligned}
$$


Repeating the latter identities for $f_{i}=\mathbb{1}_{B_{2}^{n}}$ and $\bar{f}_{i}=\mathbb{1}_{D_{n}}, i=1, \ldots, k$ and applying Theorem 4.1 gives the desired inequality for $p \geqslant 1$. Next, for $p>0$, let

$$
F\left(x_{1}, \ldots, x_{k}\right)=\left|\operatorname{conv}\left\{0, x_{1}, \ldots, x_{k}\right\}\right|^{p} .
$$

Fix $x_{1}, \ldots, x_{k-1} \in \mathbb{R}^{n}$ and $\theta \in S^{n-1}$ such that $x_{1}, \ldots, x_{k-1}, \theta$ are linearly independent. Then

$$
\mathbb{R}^{+} \ni r \mapsto F\left(x_{1}, \ldots, x_{k-1}, r \theta\right)
$$

is strictly increasing (cf. (4.8)). For $i=1, \ldots, k$, let $\bar{f}_{i *}^{*}=\left(\bar{f}_{i}\right)^{*}$. One can now extend the result to $p>0$ by writing $\Delta_{p}^{0}\left(\bar{f}_{1}{ }^{*}, \ldots, \bar{f}_{k}{ }^{*}\right)$ in spherical coordinates and applying Lemma 4.3 iteratively (as in, e.g., [11, Lemma 4.3]).

Towards the equality cases, assume that equality holds in (4.10). It follows from (4.13) and (4.14) that

$$
\Delta_{p}^{0}\left(\bar{f}_{1}, \ldots, \bar{f}_{k}\right)=\Delta_{p}^{0}\left(\mathbb{1}_{D_{n}}, \ldots, \mathbb{1}_{D_{n}}\right) .
$$

Furthermore, it follows from Theorem 4.1 applied to $C=\operatorname{conv}\left\{0, e_{1}, \ldots, e_{k}\right\}$, that

$$
\Delta_{p}^{0}\left(\bar{f}_{1}^{*}, \ldots, \bar{f}_{k}^{*}\right)=\Delta_{p}^{0}\left(\mathbb{1}_{D_{n}}, \ldots, \mathbb{1}_{D_{n}}\right) .
$$

We will first argue that for each $i=1, \ldots, k$, we must have $\bar{f}_{i}^{*}=\mathbb{1}_{D_{n}}$ a.e. Suppose towards a contradiction that the latter does not hold. Without loss of generality, we may assume that

$$
\left|\left\{x \in \mathbb{R}^{n}: \bar{f}_{k}^{*}(x) \neq \mathbb{1}_{D_{n}}(x)\right\}\right|>0 .
$$

Then $h(r):=\bar{f}_{k}^{*}(r \theta)(r>0)$ is independent of $\theta \in S^{n-1}$ and $h$ differs from $\mathbb{1}_{\left[0, r_{n}\right]}$ on a subset of positive measure. The equality condition in Lemma 4.3 implies

$$
\int_{0}^{\infty} F\left(x_{1}, \ldots, x_{k-1}, r \theta\right) h(r) r^{n-1} d r>\int_{0}^{r_{n}} F\left(x_{1}, \ldots, x_{k-1}, r \theta\right) r^{n-1} d r .
$$

Integrating in $\theta \in S^{n-1}$,

$$
\begin{aligned}
\int_{S^{n-1}} & \int_{0}^{\infty} F\left(x_{1}, \ldots, x_{k-1}, r \theta\right) \bar{f}_{k}^{*}(r \theta) r^{n-1} d r d \sigma(\theta) \\
> & \int_{S^{n-1}} \int_{0}^{r_{n}} F\left(x_{1}, \ldots, x_{k-1}, r \theta\right) r^{n-1} d r d \sigma(\theta) .
\end{aligned}
$$


In other words, for linearly independent $x_{1}, \ldots, x_{k-1}$, we have

$$
\int_{\mathbb{R}^{n}} F\left(x_{1}, \ldots, x_{k-1}, x_{k}\right)\left(\bar{f}_{k}\right)^{*}\left(x_{k}\right) d x_{k}>\int_{D_{n}} F\left(x_{1}, \ldots, x_{k-1}, x_{k}\right) d x_{k} .
$$

By continuity of $F$, we have

$$
\Delta_{p}^{0}\left(\bar{f}_{1}^{*}, \ldots, \bar{f}_{k}^{*}\right)>\Delta_{p}^{0}\left(\mathbb{1}_{D_{n}}, \ldots, \mathbb{1}_{D_{n}}\right),
$$

which contradicts (4.16).

Thus we have shown that for each $i=1, \ldots, k, \bar{f}_{i}^{*}=\mathbb{1}_{D_{n}}$ a.e. It follows that $\bar{f}_{i}=\mathbb{1}_{K_{i}}$, where $K_{i}$ is a measurable set of volume one, for $i=1, \ldots, k$. Since each set $\left\{f_{i}=\left\|f_{i}\right\|_{\infty}\right\}$ is bounded, so too is each $K_{i}$. Thus we have reduced the equality cases in (4.10) to that of bounded, Borel measurable sets and we appeal to the work of Gardner [17, Corollary 4.2] which draws on Pfiefer [36], [37]; we note that the latter articles state the equality conditions under the assumption that $K_{1}=\ldots=K_{k}$, although it is explained in [36, pgs 69-70] that the same techniques apply when the bodies $K_{i}$ are not necessarily the same.

The case $p<0$ is proved in the same way.

Another variant of Theorem 4.1 is the following result.

Theorem 4.4. Let $k$ and $n$ be positive integers. Let $f$ be a non-negative bounded, integrable function on $\mathbb{R}^{n}$ with $\|f\|_{1}>0$. Let $C \subset \mathbb{R}^{k}$ be a compact convex set and let $p \geqslant 1$. Set $m=\min (k, n, \operatorname{dim} C)$. Then

$$
\mathcal{F}_{C, p}(f, \ldots, f) \geqslant\left(\frac{\|f\|_{1}}{\omega_{n}\|f\|_{\infty}}\right)^{m / n} \mathcal{F}_{C, p}\left(\mathbb{1}_{B_{2}^{n}}, \ldots, \mathbb{1}_{B_{2}^{n}}\right),
$$

where the arguments in $\mathcal{F}_{C, p}(\cdot, \ldots, \cdot)$ are repeated $k$-times.

Proof. Set $a=\left(\|f\|_{1} /\|f\|_{\infty}\right)^{1 / n}$ and let

$$
\bar{f}(x)=\frac{f(a x)}{\int_{\mathbb{R}^{n}} f(a y) d y} .
$$

Then $\|\bar{f}\|_{1}=\|\bar{f}\|_{\infty}=1$. Using homogeneity property (4.7), we have

$$
\mathcal{F}_{C, p}(\bar{f}, \ldots, \bar{f})=\left(\frac{\|f\|_{\infty}}{\|f\|_{1}}\right)^{m / n} \mathcal{F}_{C, p}(f, \ldots, f) .
$$

Repeating the latter argument with $f=\mathbb{1}_{B_{2}^{n}}$ and $\bar{f}=\mathbb{1}_{D_{n}}$, and applying Theorem 4.1 gives the desired inequality. 
Corollary 4.5. Let $1 \leqslant k \leqslant n$ and let $f$ be a non-negative, bounded integrable function on $\mathbb{R}^{n}$ with $\|f\|_{1}>0$. For $p \neq 0$, set

$$
\Delta_{p}(f, \ldots, f)=\int_{\mathbb{R}^{n}} \cdots \int_{\mathbb{R}^{n}}\left|\operatorname{conv}\left\{x_{1}, \ldots, x_{k+1}\right\}\right|^{p} \prod_{i=1}^{k+1} f\left(x_{i}\right) d x_{1} \ldots d x_{k+1} .
$$

Then for $p \geqslant 1$,

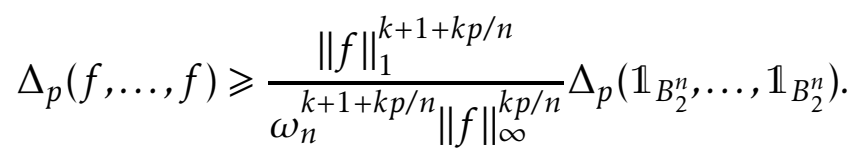

Assume additionally that $\left\{f=\|f\|_{\infty}\right\}$ is a bounded subset of $\mathbb{R}^{n}$. Then equality holds in (4.19) when $k=n$ if and only if there is an ellipsoid $\mathcal{E}$ and $a$ positive constant a such that $f=a \mathbb{1}_{\mathcal{E}}$ a.e.; when $k<n$, equality holds if and only if there is a positive constant $a$ and a Euclidean ball $B$ such that $f=a \mathbb{1}_{B}$ a.e.

The proof is parallel to that of Corollary 4.2, although the equality conditions in this case require the following additional lemma (see [33, Lemmas 3.7, 3.8]).

Lemma 4.6. Let $1 \leqslant k \leqslant n$ and let $x_{1}, \ldots, x_{k} \in \mathbb{R}^{n}$. Then for each $p \geqslant 1$,

$$
\mathbb{R}^{+} \ni r \mapsto \int_{S^{n-1}}\left|\operatorname{conv}\left\{x_{1}, \ldots, x_{k}, r \theta\right\}\right|^{p} d \sigma(\theta)
$$

is increasing.

Here and throughout, "increasing" is used in the non-strict sense.

Remark 4.7. If $k=n=1$, the condition $p \geqslant 1$ in the latter lemma is needed. Indeed, in this case $S^{0}=\{-1,1\}$ and the function $\mathbb{R}^{+} \ni r \mapsto$ $\frac{1}{2}\left(\left|r-x_{1}\right|^{p}+\left|r+x_{1}\right|^{p}\right)$ is not monotone if $p<1, p \neq 0$ and $x_{1} \neq 0$.

Proof of Corollary 4.5. Set $C=\operatorname{conv}\left\{e_{1}, \ldots, e_{k+1}\right\} \subset \mathbb{R}^{k+1}$ so that $\operatorname{dim} C=k$. Observe that

$$
\Delta_{p}(f, \ldots, f)=\|f\|_{1}^{k+1} \mathcal{F}_{C, p}(f, \ldots, f)^{p},
$$

where the arguments in $\mathcal{F}_{C, p}(\cdot, \ldots, \cdot)$ are repeated $k+1$ times. The inequality follows from Theorem 4.4 (with $k+1$ in place of $k$ and $m=k$ ).

Assume now that equality holds in (4.19). It follows that

$$
\Delta_{p}(\bar{f}, \ldots, \bar{f})=\Delta_{p}\left(\mathbb{1}_{D_{n}}, \ldots, \mathbb{1}_{D_{n}}\right),
$$


where $\bar{f}$ is defined in (4.17). In turn, we must have equality in both inequalities in Theorem 4.1. In particular,

$$
\Delta_{p}\left(\bar{f}^{*}, \ldots, \bar{f}^{*}\right)=\Delta_{p}\left(\mathbb{1}_{D_{n}}, \ldots, \mathbb{1}_{D_{n}}\right),
$$

where $\bar{f}^{*}:=(\bar{f})^{*}$. As above, we claim that $\bar{f}^{*}=\mathbb{1}_{D_{n}}$ a.e. For a contradiction, we assume that

$$
\left|\left\{x \in \mathbb{R}^{n}: \bar{f}^{*}(x) \neq \mathbb{1}_{D_{n}}(x)\right\}\right|>0 .
$$

Let

$$
F\left(x_{1}, \ldots, x_{k+1}\right)=\left|\operatorname{conv}\left\{x_{1}, \ldots, x_{k+1}\right\}\right|^{p} .
$$

By Lemma 4.6 , for any $x_{1}, \ldots, x_{k} \in \mathbb{R}^{n}$, the function

$$
\mathbb{R}^{+} \ni r \mapsto \int_{S^{n-1}} F\left(x_{1}, \ldots, x_{k}, r \theta\right) d \sigma(\theta)
$$

is increasing. By Lemma 4.3, we have

$$
\begin{aligned}
\int_{0}^{\infty} & \int_{S^{n-1}} F\left(x_{1}, \ldots, x_{k}, r \theta\right) \bar{f}^{*}(r \theta) r^{n-1} d \sigma(\theta) d r \\
& \geqslant \int_{0}^{r_{n}} \int_{S^{n-1}} F\left(x_{1}, \ldots, x_{k}, r \theta\right) r^{n-1} d \sigma(\theta) d r .
\end{aligned}
$$

i.e.

$$
\int_{\mathbb{R}^{n}} F\left(x_{1}, \ldots, x_{k}, x_{k+1}\right) \bar{f}^{*}\left(x_{k+1}\right) d x_{k+1} \geqslant \int_{D_{n}} F\left(x_{1}, \ldots, x_{k}, x_{k+1}\right) d x_{k+1} .
$$

Assume now that $x_{1}, \ldots, x_{k}$ are affinely independent points inside the support of $\bar{f}^{*}$, and $0 \in \operatorname{conv}\left\{x_{1}, \ldots, x_{k}\right\}$. Then

$$
F\left(x_{1}, \ldots, x_{k}, r \theta\right)^{1 / p}=(1 / k)\left|\operatorname{conv}\left\{x_{1}, \ldots, x_{k}\right\} \| P_{E}(r \theta)\right|,
$$

where $E=\operatorname{span}\left\{x_{1}, \ldots, x_{k}\right\}^{\perp}$. In particular, for such fixed $x_{1}, \ldots, x_{k}, F$ is a strictly increasing function of $r$. Consequenlty, for such $x_{i}$ the inequality in (4.23) is strict by Lemma 4.3. By continuity of $F$, and another application of Theorem 4.4 , we get

$$
\Delta_{p}\left(\bar{f}^{*}, \ldots, \bar{f}^{*}, \bar{f}^{*}\right)>\Delta_{p}\left(\bar{f}^{*}, \ldots, \bar{f}^{*}, \mathbb{1}_{D_{n}}\right) \geqslant \Delta_{p}\left(\mathbb{1}_{D_{n}}, \ldots, \mathbb{1}_{D_{n}}\right),
$$

which contradicts $(4.22)$.

Thus $\bar{f}^{*}=\mathbb{1}_{D_{n}}$ a.e., hence $\bar{f}=\mathbb{1}_{K}$, for some measurable set $K$ of volume one. Arguing as in the proof of Corollary 4.2, we reduce the equality case in (4.19) to that of bounded Borel measurable sets and we appeal again to the work of Gardner [17, Corollary 4.2] and Pfiefer [36], [37]. 


\section{Integral inequalities on $G_{n, k}$ and $M_{n, k}$}

In this section we prove Theorems 1.2 and 1.3. We start with a generalization of the former.

Theorem 5.1. Let $1 \leqslant q \leqslant k \leqslant n-1$ and let $f_{1}, \ldots, f_{q}$ be non-negative, bounded integrable functions on $\mathbb{R}^{n}$. Then for $0 \leqslant p \leqslant n-k$,

$$
\int_{G_{n, k}} \prod_{i=1}^{q} \frac{\left\|\left.f_{i}\right|_{E}\right\|_{1}^{1+p / k}}{\left\|\left.f_{i}\right|_{E}\right\|_{\infty}^{p / k}} d E \leqslant \frac{\omega_{k}^{q(k+p) / k}}{\omega_{n}^{q(k+p) / n}} \prod_{i=1}^{q}\left\|f_{i}\right\|_{1}^{(k+p) / n}\left\|f_{i}\right\|_{\infty}^{(n-k-p) / n} .
$$

In particular, when $q=k$ and $p=n-k$, we have

$$
\int_{G_{n, k}} \prod_{i=1}^{k} \frac{\left\|\left.f_{i}\right|_{E}\right\|_{1}^{n / k}}{\left\|\left.f_{i}\right|_{E}\right\|_{\infty}^{(n-k) / k}} d E \leqslant \frac{\omega_{k}^{n}}{\omega_{n}^{k}} \prod_{i=1}^{k}\left\|f_{i}\right\|_{1} .
$$

Assume additionally that $\left\{f_{i}=\left\|f_{i}\right\|_{\infty}\right\}$ is bounded and $f_{i}$ is continuous at 0 for each $i=1, \ldots, k$ and $p>0$. Then equality holds in (5.1) for $k=1$ if and only if $f_{1}=a \mathbb{1}_{K}$ a.e., where $a>0$ and $K \subset \mathbb{R}^{n}$ is star-shaped about the origin; for $1 \leqslant q<k$ or $0<p<n-k$ if and only if there are positive constants $a_{i}$, $b_{i}$, such that $f_{i}=a_{i} \mathbb{1}_{b_{i} B_{2}^{n}}$ a.e.; equality holds in (5.2) if and only if there is an origin-symmetric ellipsoid $\mathcal{E} \subset \mathbb{R}^{n}$ and positive constants $a_{i}, b_{i}$, such that $f_{i}=a_{i} \mathbb{1}_{b_{i} \mathcal{E}}$ a.e. for $i=1, \ldots, k$.

Proof of Theorem 5.1. We will use the following well-known identity from integral geometry: for $p>-(k-q+1)$, we have

$$
\Delta_{-(n-k-p)}^{0}\left(f_{1}, \ldots, f_{q}\right)=c_{n, k, q} \int_{G_{n, k}} \Delta_{p}^{0}\left(\left.f_{1}\right|_{E}, \ldots,\left.f_{q}\right|_{E}\right) d E,
$$

where $\Delta_{p}^{0}$ is defined in (4.9); this is simply Theorem 2.1 applied to the function

$$
F\left(x_{1}, \ldots, x_{q}\right)=\prod_{i=1}^{q} f_{i}\left(x_{i}\right)\left|\operatorname{conv}\left\{0, x_{1}, \ldots, x_{q}\right\}\right|^{-(n-k-p)} .
$$

Assume now that $E \in G_{n, k}$ and $\left\|\left.f_{i}\right|_{E}\right\|_{\infty}>0$ for $i=1, \ldots, q$. Applying Corollary 4.2 on $E$ (with $k$ in place of $n$ and $q$ in place of $k$ ), we have

$$
\prod_{i=1}^{q} \frac{\left\|\left.f_{i}\right|_{E}\right\|_{1}^{1+p / k}}{\left\|\left.f_{i}\right|_{E}\right\|_{\infty}^{p / k}} \leqslant \omega_{k}^{q(k+p) / k} \frac{\Delta_{p}^{0}\left(\left.f_{1}\right|_{E}, \ldots,\left.f_{q}\right|_{E}\right)}{\Delta_{p}^{0}\left(\mathbb{1}_{B_{2}^{n} \cap E}, \ldots, \mathbb{1}_{B_{2}^{n} \cap E}\right)} .
$$


Note that $\Delta_{p}^{0}\left(\mathbb{1}_{B_{2}^{n} \cap E}, \ldots, \mathbb{1}_{B_{2}^{n} \cap E}\right)$ is independent of $E$. Thus, integrating over $G_{n, k}$, using (5.3) and Corollary 4.2 once more, we have

$$
\begin{aligned}
\int_{G_{n, k}} \prod_{i=1}^{q} \frac{\left\|\left.f_{i}\right|_{E}\right\|_{1}^{1+p / k}}{\left\|\left.f_{i}\right|_{E}\right\|_{\infty}^{p / k}} d E & \leqslant \omega_{k}^{q(k+p) / k} \frac{\int_{G_{n, k}} \Delta_{p}^{0}\left(\left.f_{1}\right|_{E}, \ldots,\left.f_{q}\right|_{E}\right) d E}{\int_{G_{n, k}} \Delta_{p}^{0}\left(\mathbb{1}_{B_{2}^{n} \cap E}, \ldots, \mathbb{1}_{B_{2}^{n} \cap E}\right) d E} \\
& =\omega_{k}^{q(k+p) / k} \frac{\Delta_{-(n-k-p)}^{0}\left(f_{1}, \ldots, f_{q}\right)}{\Delta_{-(n-k-p)}^{0}\left(\mathbb{1}_{B_{2}^{n}}, \ldots, \mathbb{1}_{\left.B_{2}^{n}\right)}\right.} \\
& \leqslant \frac{\omega_{k}^{q(k+p) / k}}{\omega_{n}^{q(k+p) / n}} \prod_{i=1}^{q}\left\|f_{i}\right\|_{1}^{(k+p) / n}\left\|f_{i}\right\|_{\infty}^{(n-k-p) / n}
\end{aligned}
$$

The equality cases follow from those of Corollary 4.2 and Gardner's characterizations of sets that are ellipsoids, Euclidean balls or star-shaped, up to sets of measure zero, in [17, Section 6].

Theorem 5.2. Let $1 \leqslant k \leqslant n-1$ and $f$ be a non-negative bounded integrable function on $\mathbb{R}^{n}$. Then

$$
\int_{M_{n, k}} \frac{\left(\int_{F} f(x) d x\right)^{n+1}}{\left\|\left.f\right|_{F}\right\|_{\infty}^{n-k}} d F \leqslant \frac{\omega_{k}^{n+1} \omega_{n(k+1)}}{\omega_{n}^{k+1} \omega_{k(n+1)}}\left(\int_{\mathbb{R}^{n}} f(x) d x\right)^{k+1} .
$$

Assume additionally that $\left\{f=\|f\|_{\infty}\right\}$ is bounded. Then equality holds in (5.8) when $k=1$ if and only if $f=\mathbb{1}_{K}$, where $K \subset \mathbb{R}^{n}$ is a convex body; when $k>1$ if and only if there a positive constant $a$ and an ellipsoid $\mathcal{E}$ such that $f=a \mathbb{1}_{\mathcal{E}}$ a.e.

When $f=\mathbb{1}_{K}$, where $K$ is a convex body in $\mathbb{R}^{n}$, a more general result is due to Schneider [41]: for $s \in\{1, \ldots, n\}$,

$$
\int_{M_{n, k}}|K \cap F|^{s+1} d F \leqslant \frac{\omega_{k}^{s+1}}{\omega_{n}^{(n+k s) / n}} \frac{\omega_{n+k s}}{\omega_{k+k s}}|K|^{1+k s / n} .
$$

It is natural to try to extend Theorem 5.2 to powers $1 \leqslant s \leqslant n$ as in (5.9). Following the line of proof of Theorem 5.1 would require a statement such as Corollary 4.5 for $p<1$ (in particular $p<0$ ); it is unclear to us if this is possible.

Proof of Theorem 5.2. Let $F \in M_{n, k}$ and assume $\left\|\left.f\right|_{F}\right\|_{\infty}>0$. Applying 
Corollary 4.5 on $F$ with $p=n-k$ (replacing $n$ by $k$ ) we have

$$
\frac{\left(\int_{F} f(x) d x\right)^{n+1}}{\left\|\left.f\right|_{F}\right\|_{\infty}^{n-k}} \leqslant \omega_{k}^{n+1} \frac{\Delta_{n-k}\left(\left.f\right|_{F}, \ldots,\left.f\right|_{F}\right)}{\Delta_{n-k}\left(\mathbb{1}_{B_{2}^{k}}, \ldots, \mathbb{1}_{B_{2}^{k}}\right)},
$$

where the arguments in $\Delta_{n-k}$ are repeated $k+1$ times. Integrating over $M_{n, k}$ and applying Theorem 2.2, we get

$$
\begin{aligned}
\int_{M_{n, k}} \frac{\left(\int_{F} f(x) d x\right)^{n+1}}{\left\|\left.f\right|_{F}\right\|_{\infty}^{n-k}} d F & \leqslant \frac{\omega_{k}^{n+1} \int_{M_{n, k}} \Delta_{n-k}\left(\left.f_{1}\right|_{F}, \ldots,\left.f_{k+1}\right|_{F}\right) d F}{\Delta_{n-k}\left(\mathbb{1}_{B_{2}^{k}}, \ldots, \mathbb{1}_{B_{2}^{k}}\right)} \\
& =\frac{\omega_{k}^{n+1}}{c_{n, k, k} \Delta_{n-k}\left(\mathbb{1}_{B_{2}^{k}}, \ldots, \mathbb{1}_{B_{2}^{k}}\right)}\left(\int_{\mathbb{R}^{n}} f(x) d x\right)^{k+1} .
\end{aligned}
$$

If $f=\mathbb{1}_{B_{2}^{n}}$, then inequality (5.10) is an equality (as noted in [41]), hence so is (5.11). Consequently, using the expression in the equality case in (1.12) and rearranging terms, we get

$$
\Delta_{n-k}\left(\mathbb{1}_{B_{2}^{k}}, \ldots, \mathbb{1}_{B_{2}^{k}}\right)=c_{n, k, k} \omega_{n}^{k+1} \frac{\omega_{k(n+1)}}{\omega_{n(k+1)}},
$$

The latter also follows from results of Kingman [22] and Miles [28]. This proves the inequality.

The equality cases follow from those of Corollary 4.5 and [17, Corollary 6.8].

\section{Bounds for marginals}

In this section we state and prove a generalization of Theorem 1.1. We also discuss marginals of log-concave measures and connections to the Hyperplane Conjecture.

Theorem 6.1. Suppose $\mu$ is a probability measure on $\mathbb{R}^{n}$ with a bounded density $f$. Then for each $1 \leqslant k \leqslant n-1$ and $s>1$, there exists $\mathcal{A}_{s} \subseteq G_{n, k}$ with $\mu_{n, k}\left(\mathcal{A}_{s}\right) \geqslant 1-2 s^{-k n}$ such that:

(i) for every $E \in \mathcal{A}_{s}$ and $t>1$, there exists a set $\mathcal{B}_{t} \subseteq E$ such that $\pi_{E}(\mu)\left(\mathcal{B}_{t}\right) \leqslant$ $t^{-k n}$ and

$$
f_{\pi_{E}(\mu)}(x)^{1 / k} \leqslant c_{1} s t\|f\|_{\infty}^{1 / n}, \quad\left(x \in\left(E \backslash \mathcal{B}_{t}\right) \cup\{0\}\right) ;
$$


(ii) for every $E \in \mathcal{A}_{s}, \varepsilon>0$ and any $z \in E$,

$$
\pi_{E}(\mu)(\{x \in E:|x-z| \leqslant \varepsilon \sqrt{k}\}) \leqslant\left(c_{2} s \varepsilon\|f\|_{\infty}^{1 / n}\right)^{k n /(n+1)} .
$$

Proof. By Fubini's Theorem and Theorem 1.3,

$$
\begin{aligned}
\left(\int_{G_{n, k}} \int_{E} \frac{\left(\int_{E^{\perp}+x} f(y) d y\right)^{n}}{\left\|\left.f\right|_{E^{\perp}+x}\right\|_{\infty}^{k}} d \pi_{E}(\mu)(x) d E\right)^{\frac{1}{k n}} & =\left(\int_{M_{n, n-k}} \frac{\left(\int_{F} f(y) d y\right)^{n+1}}{\left\|\left.f\right|_{F}\right\|_{\infty}^{k}} d F\right)^{\frac{1}{k n}} \\
& \leqslant\left(\frac{\omega_{n-k}^{n+1} \omega_{n(n-k+1)}}{\omega_{n}^{n-k+1} \omega_{(n-k)(n+1)}}\right)^{\frac{1}{k n}} \\
& \simeq 1 .
\end{aligned}
$$

By Markov's inequality, for each $s>1$, the $\mu_{n, k}$-measure of the set $\mathcal{A}_{s}^{(1)}$ of $E \in G_{n, k}$ such that

$$
\int_{E} \frac{\left(\int_{E^{\perp}+x} f(y) d y\right)^{n}}{\left\|\left.f\right|_{E^{\perp}+x}\right\|_{\infty}^{k}} d \pi_{E}(\mu)(x) \leqslant(c s)^{k n}
$$

is at least $1-s^{-k n}$. For $E \in \mathcal{A}_{s}^{(1)}$ and $t>1$, we apply Markov's inequality once more to get that the $\pi_{E}(\mu)$-measure of the set $\mathcal{B}_{t}$ of $x \in E$ such that

$$
\frac{\left(\int_{E^{\perp}+x} f(y) d y\right)^{n}}{\left\|\left.f\right|_{E^{\perp}+x}\right\|_{\infty}^{k}} \geqslant(c s t)^{k n}
$$

is less than $t^{-k n}$. Note that for every $x \in E \backslash \mathcal{B}_{t}$,

$$
\left(\int_{E^{\perp}+x} f(y) d y\right)^{1 / k} \leqslant c s t\left\|\left.f\right|_{E^{\perp}+x}\right\|_{\infty}^{1 / n} \leqslant c s t\|f\|_{\infty}^{1 / n} .
$$

Applying now Theorem 1.2, we have

$$
\left(\int_{G_{n, k}} \frac{f_{\pi_{E}(\mu)}(0)^{n}}{\left\|\left.f\right|_{E^{\perp}}\right\|_{\infty}^{k}} d E\right)^{\frac{1}{n k}}=\left(\int_{G_{n, k}} \frac{\left(\int_{E^{\perp}} f(y) d y\right)^{n}}{\left\|\left.f\right|_{E^{\perp}}\right\|_{\infty}^{k}} d E\right)^{\frac{1}{n k}} \leqslant\left(\frac{\omega_{n-k}^{n}}{\omega_{n}^{n-k}}\right)^{\frac{1}{n k}} \simeq 1 .
$$

By Markov's inequality, for each $s>1$, the $\mu_{n, k}$-measure of the set $\mathcal{A}_{s}^{(2)}$ of $E \in G_{n, k}$ such that

$$
f_{\pi_{E}(\mu)}(0)^{\frac{1}{k}} \leqslant c s\left\|\left.f\right|_{E^{\perp}}\right\|_{\infty}^{\frac{1}{n}}
$$


is at least $1-s^{-n k}$. For every $E \in \mathcal{A}_{s}^{(2)}$, we have that

$$
f_{\pi_{E}(\mu)}(0)^{\frac{1}{k}} \leqslant c s\left\|\left.f\right|_{E^{\perp}}\right\|_{\infty}^{\frac{1}{n}} \leqslant c s\|f\|_{\infty}^{\frac{1}{n}}
$$

Setting $\mathcal{A}_{s}:=\mathcal{A}_{s}^{1} \cup \mathcal{A}_{s}^{(2)}$, we conlude that (6.1) holds.

Towards (6.2), let $s, t>1, E \in \mathcal{A}_{s}, z \in E$ and $\varepsilon>0$. Then

$$
\begin{aligned}
\pi_{E}( & \mu)(\{x \in E:|x-z| \leqslant \varepsilon \sqrt{k}\}) \\
= & \int_{B(z, \varepsilon \sqrt{k})} f_{\pi_{E}(\mu)}(y) d y \\
& =\int_{B(z, \varepsilon \sqrt{k}) \cap \mathcal{B}_{t}} f_{\pi_{E}(\mu)}(y) d y+\int_{B(z, \varepsilon \sqrt{k}) \cap \mathcal{B}_{t}^{c}} f_{\pi_{E}(\mu)}(y) d y \\
& =\pi_{E}(\mu)\left(\mathcal{B}_{t}\right)+\sup _{y \in E \backslash \mathcal{B}_{t}} f_{\pi_{E}(\mu)}(y)|B(z, \varepsilon \sqrt{k})| \\
& \leqslant t^{-n k}+\left(c_{1} s t\|f\|_{\infty}^{1 / n}\right)^{k}\left(c_{0} \varepsilon\right)^{k} .
\end{aligned}
$$

We choose $t:=\left(c_{2} \varepsilon s\|f\|_{\infty}^{1 / n}\right)^{-\frac{1}{n+1}}$ and we get (6.2).

Remark 6.2. If $\mathcal{A}_{s}$ is the set in Theorem 1.1, $E \in \mathcal{A}_{s}$ and $\varepsilon>0$, then we have the stronger small-ball probability

$$
\pi_{E}(\mu)(\{x \in E:|x| \leqslant \varepsilon \sqrt{k}\}) \leqslant\left(c_{3} s \varepsilon\|f\|_{\infty}^{1 / n}\right)^{k} .
$$

The proof is analogous to that of (6.2) (use Theorem 1.2).

The next lemma shows that the probability estimate for the $\mu_{n, k^{-}}$ measure in Theorem 6.1 is sharp in each dimension $k$.

Lemma 6.3. Let $1 \leqslant k \leqslant n-1$ and set $\sigma=(2 \pi)^{-n /(2 k)}$. Let $f$ be the Gaussian density with law $\mu=N(0, D)$, where $D$ is the diagonal matrix $D=$ $\operatorname{diag}\left(\sigma^{2}, \ldots, \sigma^{2}, 1, \ldots, 1\right)$, with $\sigma^{2}$ repeated $k$ times. Then for each $1 \leqslant s \leqslant \sigma^{-1}$,

$$
\mu_{n, k}\left(\left\{E \in G_{n, k}:\left\|f_{\pi_{E}(\mu)}\right\|_{\infty}^{1 / k} \geqslant s\right\}\right) \geqslant(2 s)^{-k(n-k)} .
$$

The proof relies on the following proposition, which is a direct consequence of a result of Szarek [43]; this formulation is from [35, Corollary 2.2]; here we equip $G_{n, k}$ with the metric $d\left(E_{0}, E_{1}\right)$ which is the operator norm $\left\|P_{E_{0}}-P_{E_{1}}: \ell_{2}^{n} \rightarrow \ell_{2}^{n}\right\|$. 
Proposition 6.4. Let $1 \leqslant k \leqslant n-1, E \in G_{n, k}$ and $\varepsilon \in(0,2)$. Then

$$
\mu_{n, k}\left(\left\{F \in G_{n, k}: d(E, F) \leqslant \varepsilon\right\}\right) \geqslant(c \varepsilon)^{k(n-k)},
$$

where $c$ is a numeric constant.

Proof of Lemma 6.3. Let $g$ be a standard Gaussian vector in $\mathbb{R}^{n}$. Let $E_{0}$ be the span of first $k$ coordinate unit vectors and note that

$$
\|f\|_{\infty}=\left(2 \pi \sigma^{2}\right)^{-k / 2}(2 \pi)^{-(n-k) / 2}=1,
$$

while

$$
\left\|f_{\pi_{E_{0}}(\mu)}\right\|_{\infty}=(2 \pi)^{(n-k) / 2} .
$$

Let $\varepsilon>0$ and assume that $E_{1} \in G_{n, k}$ satisfies $d\left(E_{0}, E_{1}\right)<\varepsilon$. Write

$$
P_{E_{1}} D=\sigma P_{E_{1}} P_{E_{0}}+P_{E_{1}} P_{E_{0}^{\perp}} \text {. }
$$

Using singular value decomposition, there exist orthonormal bases $u_{1}, \ldots, u_{k}$ of $E_{0}$ and $v_{1}, \ldots, v_{k}$ of $E_{1}$ such that

$$
P_{E_{1}} P_{E_{0}}=\sum_{i=1}^{k} a_{i} u_{i} \otimes v_{i}
$$

where $0 \leqslant a_{i}=\left\langle u_{i}, v_{i}\right\rangle \leqslant 1$. Since $P_{E_{1}} P_{E_{0}^{\perp}}=P_{E_{1}}\left(I-P_{E_{0}}\right)$, we can write

$$
P_{E_{1}} P_{E_{0}^{\perp}}=\sum_{i=1}^{k} v_{i} \otimes v_{i}-\sum_{i=1}^{k} a_{i} u_{i} \otimes v_{i}=\sum_{i: a_{i} \neq 1} \sqrt{1-a_{i}^{2}} f_{i} \otimes v_{i},
$$

where $f_{i}=\frac{v_{i}-a_{i} u_{i}}{\sqrt{1-a_{i}^{2}}}$. Set $\gamma_{i}=\left\langle g, u_{i}\right\rangle$ and $\gamma_{i}^{\prime}=\left\langle g, f_{i}\right\rangle$. Since $u_{i}$ and $f_{i}$ are orthogonal, $\gamma_{i}$ and $\gamma_{i}^{\prime}$ are independent. Note that

$$
P_{E_{1}} D g=\sum_{i: a_{i}=1} \sigma \gamma_{i} v_{i}+\sum_{i: a_{i} \neq 1}\left(\sigma a_{i} \gamma_{i}+\sqrt{1-a_{i}^{2}} \gamma_{i}^{\prime}\right) v_{i}
$$

Sicne $\sqrt{1-a_{i}^{2}} \leqslant\left\|P_{E_{0}}-P_{E_{1}}\right\|=d\left(E_{0}, E_{1}\right) \leqslant \varepsilon$ for each $i=1, \ldots, k$, the covariance matrix $A$ of $P_{E_{1}} D g$ satisfies

$$
(\operatorname{det}(A))^{1 / k} \leqslant \frac{1}{k} \operatorname{tr}(A)=\frac{1}{k} \sum_{i=1}^{k}\left(\sigma^{2} a_{i}^{2}+1-a_{i}^{2}\right) \leqslant \sigma^{2}+\varepsilon^{2} .
$$


It follows that for any $E_{1}$ with $d\left(E_{0}, E_{1}\right) \leqslant \varepsilon$, we have

$$
\left\|f_{\pi_{E_{1}}(\mu)}\right\|_{\infty}^{1 / k}=\frac{1}{\left((2 \pi)^{k} \operatorname{det}(A)\right)^{1 /(2 k)}} \geqslant \frac{1}{\sqrt{2 \pi\left(\sigma^{2}+\varepsilon^{2}\right)}} .
$$

We now apply Proposition 6.4 with $\varepsilon=1 /(c s)$ to obtain (6.6).

\subsection{Concluding remarks}

In light of Theorem 6.1, a natural question arises here: under what additional condition(s), can one guarantee that all marginal densities of such functions $f$ are suitably bounded, i.e.,

$$
\left\|f_{\pi_{E}(\mu)}\right\|_{\infty}^{\frac{1}{k}} \leqslant C\|f\|_{\infty}^{\frac{1}{n}}, \quad \forall E \in G_{n, k}
$$

as in the case of Rudelson and Vershynin's result (1.2). The example of independent Gaussians with different variances in Lemma 6.3 shows that one needs a type of non-degeneracy condition. For instance, one may assume that $\mu$ is isotropic, namely,

$$
\int_{\mathbb{R}^{n}}\langle x, \theta\rangle d \mu(x)=0 \text { and } \int_{\mathbb{R}^{n}}|\langle x, \theta\rangle|^{2} d \mu(x)=1 \quad \forall \theta \in S^{n-1} .
$$

If $\mu$ is an isotropic, subgaussian, log-concave probability measure then (6.7) holds as a consequence of a result of Bourgain on the isotropic constant of such measures [4]. If $\mu$ is isotropic and log-concave, the isotropic constant of $\mu$ is defined by $L_{\mu}:=\left\|f_{\mu}\right\|_{\infty}^{1 / n}$. A major open problem known as the Hyperplane Conjecture asks if there exists an absolute constant $C$ (independent of $n$ and $\mu$ ) such that $L_{\mu} \leqslant C$. The best known bound (of order $n^{1 / 4}$ ) is due to Klartag [23], improving an earlier result of Bourgain [3]. For detailed discussion on this conjecture, see [6]. Thus in the class of isotropic log-concave probability measures $\mu$, inequality (6.7) amounts to asking if

$$
L_{\pi_{E}(\mu)} \leqslant C L_{\mu}, \forall E \in G_{n, k} .
$$

It is not difficult to show that the above question is just another equivalent formulation of the Hyperplane Conjecture. (For a proof of this fact see [32]). The inequality of Busemann-Straus and Grinberg (1.11) has been used recently in [35] to show that the marginals that satisfy the Hyperplane Conjecture form a 1 -net in $G_{n, k}$ for $k \leqslant \sqrt{n}$. One of the main 
ingredients in the proof is entropy numbers on the Grassmanian established by Szarek (Proposition 6.4 above). Using these estimates along with Theorem 1.1, we get the following corollary.

Corollary 6.5. Let $\mu$ be a probability on $\mathbb{R}^{n}$ with a bounded density $f$. Then for every $1 \leqslant k \leqslant n-1, E \in G_{n, k}$ and $\eta>0$, there exists $E_{0} \in G_{n, k}$ with $d\left(E_{0}, E\right) \leqslant \eta$ such that for any $z \in E_{0}$,

$$
\pi_{E_{0}}(\mu)\left(\left\{x \in E_{0}:|x-z| \leqslant \varepsilon \sqrt{k}\right\}\right) \leqslant\left(c_{2} \frac{\varepsilon}{\eta}\|f\|_{\infty}^{1 / n}\right)^{\frac{k n}{n+1}} .
$$

In other words, given any $E \in G_{n, k}$, there exists $E_{0}$, close to $E$ such that $E_{0}$ has a nearly optimal small-ball probability estimate.

\section{Acknowledgements}

It is our pleasure to thank Alex Koldobsky, Fedja Nazarov, Gestur Olafsson and Petros Valettas for helpful discussions. The first-named author thanks the Oberwolfach Research Institute for Mathematics for its hospitality and support, where part of this work was carried out. The second-named author acknowledges with thanks support from the A. Sloan foundation, BSF grant 2010288 and the US NSF grant CAREER1151711. The third-named author would like to thank the University of Missouri Research Board and Simons Foundation (grant \#317733) for financial support which facilitated our collaboration.

\section{References}

[1] A. Baernstein, II and M. Loss, Some conjectures about $L^{p}$ norms of $k$-plane transforms, Rend. Sem. Mat. Fis. Milano 67 (1997), 9-26 (2000).

[2] C. Bishop and Y. Peres, Fractal sets in probability and analysis, Book in preparation.

[3] J. Bourgain, On the distribution of polynomials on high-dimensional convex sets, Geometric aspects of functional analysis (1989-90), Lecture Notes in Math., vol. 1469, Springer, Berlin, 1991, pp. 127-137.

[4] On the isotropy-constant problem for "PSI-2"-bodies, Geometric aspects of functional analysis, Lecture Notes in Math., vol. 1807, Springer, Berlin, 2003, pp. 114-121.

[5] H. J. Brascamp, Elliott H. Lieb, and J. M. Luttinger, A general rearrangement inequality for multiple integrals, J. Functional Analysis 17 (1974), 227-237.

[6] S. Brazitikos, A. Giannopoulos, P. Valettas, and B. H. Vritsiou, Geometry of isotropic convex bodies, Mathematical Surveys and Monographs, vol. 196, American Mathematical Society, Providence, RI, 2014.

[7] H. Busemann, Volume in terms of concurrent cross-sections, Pacific J. Math. 3 (1953), 1-12. 
[8] H. Busemann and E. G. Straus, Area and normality, Pacific J. Math. 10 (1960), 35-72.

[9] M. Christ, Extremizers of a radon transform inequality, arxiv . org/abs/1106.0719.

[10] Estimates for the k-plane transform, Indiana Univ. Math. J. 33 (1984), no. 6, 891-910.

[11] E. Cordero-Erausquin, M. Fradelizi, G. Paouris, and P Pivovarov, On the volume of the polar of random sets and shadow systems, to appear in Math. Ann., DOI 10.1007/s00208-014-1156$\mathrm{x}$.

[12] A. Druout, Best constant and value of extremizers for a $k$-plane transform inequality, arxiv.org/abs/1111.5061.

[13] S. W. Drury, Generalizations of Riesz potentials and $L^{p}$ estimates for certain $k$-plane transforms, Illinois J. Math. 28 (1984), no. 3, 495-512.

[14] L. C. Evans and R. F. Gariepy, Measure theory and fine properties of functions, Studies in Advanced Mathematics, CRC Press, Boca Raton, FL, 1992.

[15] T. Flock, Uniqueness of extremizers for an endpoint inequality of the $k$-plane transform, arxiv.org/abs/1307.6551.

[16] H. Furstenberg and I. Tzkoni, Spherical functions and integral geometry, Israel J. Math. 10 (1971), 327-338.

[17] R. J. Gardner, The dual Brunn-Minkowski theory for bounded Borel sets: dual affine quermassintegrals and inequalities, Adv. Math. 216 (2007), no. 1, 358-386.

[18] E. L. Grinberg, Isoperimetric inequalities and identities for $k$-dimensional cross-sections of convex bodies, Math. Ann. 291 (1991), no. 1, 75-86.

[19] H. Groemer, On some mean values associated with a randomly selected simplex in a convex set, Pacific J. Math. 45 (1973), 525-533.

[20] On the mean value of the volume of a random polytope in a convex set, Arch. Math. (Basel) 25 (1974), 86-90.

[21] S. Helgason, Groups and geometric analysis, Mathematical Surveys and Monographs, vol. 83, American Mathematical Society, Providence, RI, 2000, Integral geometry, invariant differential operators, and spherical functions, Corrected reprint of the 1984 original.

[22] J. F. C. Kingman, Random secants of a convex body, J. Appl. Probability 6 (1969), 660-672.

[23] B. Klartag, On convex perturbations with a bounded isotropic constant, Geom. Funct. Anal. 16 (2006), no. 6, 1274-1290.

[24] A. W. Knapp, Representation theory of semisimple groups, Princeton Mathematical Series, vol. 36, Princeton University Press, Princeton, NJ, 1986, An overview based on examples.

[25] E. H. Lieb and M. Loss, Analysis, second ed., Graduate Studies in Mathematics, vol. 14, American Mathematical Society, Providence, RI, 2001.

[26] E. Lutwak, A general isepiphanic inequality, Proc. Amer. Math. Soc. 90 (1984), no. 3, 415421.

[27] _ Intersection bodies and dual mixed volumes, Adv. in Math. 71 (1988), no. 2, 232-261.

[28] R. E. Miles, Isotropic random simplices, Advances in Appl. Probability 3 (1971), 353-382.

[29] E. Milman, Dual mixed volumes and the slicing problem, Adv. Math. 207 (2006), no. 2, 566598.

[30] - Generalized intersection bodies, J. Funct. Anal. 240 (2006), no. 2, 530-567. 
[31] G. Ólafsson and A. Pasquale, The $\operatorname{Cos}^{\lambda}$ and $\operatorname{Sin}^{\lambda}$ transforms as intertwining operators between generalized principal series representations of $\operatorname{SL}(n+1, \mathbb{K})$, Adv. Math. 229 (2012), no. 1, 267293.

[32] G. Paouris, On the isotropic constant of marginals, Studia Math. 212 (2012), no. 3, 219-236.

[33] G. Paouris and P. Pivovarov, A probabilistic take on isoperimetric-type inequalities, Adv. Math. 230 (2012), no. 3, 1402-1422.

[34] Small-ball probabilities for the volume of random convex sets, Discrete Comput. Geom. 49 (2013), no. 3, 601-646.

[35] G. Paouris and P. Valettas, Neighborhoods on the Grassmannian of marginals with bounded isotropic constant, 267 (2014), 3427-3443.

[36] R. E. Pfiefer, The extrema of geometric mean values, ProQuest LLC, Ann Arbor, MI, 1982, Thesis (Ph.D.)-University of California, Davis.

[37] _ Maximum and minimum sets for some geometric mean values, J. Theoret. Probab. 3 (1990), no. 2, 169-179.

[38] C. A. Rogers, A single integral inequality, J. London Math. Soc. 32 (1957), 102-108.

[39] M. Rudelson and R. Vershynin, Small ball probabilities for linear images of high-dimensional distributions, to appear in Int. Math. Res. Notices; arXiv . org/abs/1402. 4492.

[40] - Non-asymptotic theory of random matrices: extreme singular values, Proceedings of the International Congress of Mathematicians. Volume III, Hindustan Book Agency, New Delhi, 2010, pp. 1576-1602.

[41] R. Schneider, Inequalities for random flats meeting a convex body, J. Appl. Probab. 22 (1985), no. 3, 710-716.

[42] R. Schneider and W. Weil, Stochastic and integral geometry, Probability and its Applications (New York), Springer-Verlag, Berlin, 2008.

[43] S. J. Szarek, The finite-dimensional basis problem with an appendix on nets of Grassmann manifolds, Acta Math. 151 (1983), no. 3-4, 153-179.

Susanna Dann: Vienna University of Technology, Wiedner Hauptstrasse 8-10, 1040 Vienna, Austria

susanna.dann@tuwien.ac.at

Grigoris Paouris: Department of Mathematics, Mailstop 3368, Texas A\&M University, College Station, TX 77843-3368, USA

grigoris@math.tamu.edu

Peter Pivovarov: Mathematics Department, University of Missouri, Columbia, MO 65211, USA, pivovarovp@missouri.edu 